Military Technical College

Kobry Elkobbah,

Cairo, Egypt

April 19-21,2016

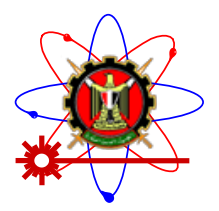

$8^{\text {th }}$ International Conference on Mathematics and Engineering Physics (ICMEP-8)

\title{
EM-4
}

\section{MHD Peristaltic Flow of Dusty Fluid through Flexible Channel under Slip Condition}

\author{
I. M. Eldesoky ${ }^{(1)}$, W. A. El-Askary ${ }^{(2)}$, A.M.El-Refaey ${ }^{(1)}$, M.M.Ahmed ${ }^{(1)}$ \\ (1) Basic Engineering Sciences Department, Faculty of Engineering, Shebin El-Kom, \\ MenoufiyaUniversity, Egypt \\ (2) Mechanical Power Engineering Department, Faculty of Engineering, Shebin El-Kom, \\ MenoufiyaUniversity, Egypt \\ islameldesoky<eldesokyi@yahoo.com>
}

\begin{abstract}
The effects of slip condition and particles concentration are investigated on the peristaltic flow of a Newtonian suspended fluid flows through a channel under different parameters. The walls of the channel are assumed flexible with an external uniform magnetic field applied perpendicular to the walls. The equations of motion are solved analytically using the perturbation method to yield the pressure, streamlines and the velocity distributions for both fluid and dusty particles. The governing equations of the flow through magnetic field are solved under the effect of concentration, wave number, amplitude ratio, Reynolds number and slip condition. The resulting relations for velocity and pressure gradient are plotted for various pertinent parameters. It was observed that in the case of positive pressure gradient, the reversal flow intensity increases with increasing the particle concentration, while it decreases with increasing the magnetic field. Furthermore, thereversal intensity increases with positive pressure gradient. For negative pressure gradient, the intensity increases with increasing the magnetic field. On the other hand, the critical reflux pressure gradient decreases with the presence of suspension and magnetic field, while the critical reflux pressure increases with slip condition. The streamlines are also computed and drawn for some physical quantities to discuss the trapping phenomenon.
\end{abstract}

Key Words:Peristaltic Transport, MHD, Dusty fluid, Flexible channel, Slip effects 
Military Technical College

Kobry Elkobbah,

Cairo, Egypt

April 19-21,2016

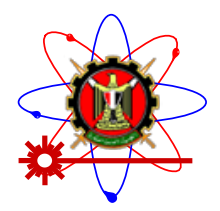

$8^{\text {th }}$ International Conference on Mathematics and Engineering Physics (ICMEP-8)

\section{Introduction}

Peristaltic pumping is a form of fluid transport that occurs when a progressive wave of area contraction or expansion propagates along the length of distensible duct containing liquid. Peristalsis is used by the body to propel or mix the contents of a tube as in ureter, swallowing food through the esophagus, movement of chyme in the gastrointestinal tract, movement of ovum in the female fallopian tube, motion of spermatozoa in cervical canal, transport of bile in bile duct, transport of lymph in the lymphatic vessels and vasomotion of small blood vessels such as arterioles, venules and capillaries.In addition, peristaltic pumping occurs in many practical applications involving biomechanical systems. Also, finger and roller pumps are frequently used for pumping corrosive or very pure materials so as to prevent direct contact of the fluid with the pumps internal surfaces. Moreover, by using the principle of peristalsis, some biomechanical instruments such as heart-lung machine, have been fabricated. The mechanism of peristaltic transporthas been exploited for industrial applications like sanitary fluid transport, blood pumps in heart lung machine, andtransport of corrosive fluids where the contact of the fluid with the machinery parts is prohibited. It is alsospeculated that peristalsis may be involved in the translocation of water in tall trees. The problem of the mechanism of peristalsistransport has attracted the attention of many investigators since the investigation of Latham [1].All important literature up to 1978 on peristaltic transport has been documented by many researchers. Fung and Yih[2] analyzed the role of Reynolds number and wavelength in peristaltic motion of moderate amplitude, making use of perturbation method with an amplitude ratio as the perturbation parameter. Shapiro et al. [3] have studied peristaltic pumping with long wavelength at low 
Military Technical College

Kobry Elkobbah,

Cairo, Egypt

April 19-21,2016

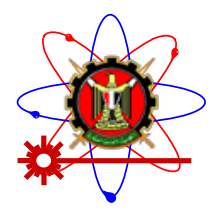

$8^{\text {th }}$ International Conference on Mathematics and Engineering Physics (ICMEP-8)

Reynolds number. After these studies,several authors[4 - 7] have studied peristalsis under different conditions.The peristaltic couple fluid flow through channels with flexible walls has been studied by Ravikumar et al. [8]. Peristaltic flow of a couple stress fluids through porous medium in a channel at low Reynolds number was studied by RaghunathaRao and Parasad [9].Mishra and RamachandraRao[10] have investigated the flow in an asymmetric channel generated by peristaltic waves propagating on the walls with different amplitudes and phases.The most physiological fluids including blood behave as nonNewtonian fluids. Hence, the study of peristaltic transport of non-Newtonian fluids may help to get better understanding of the biological systems. Several researchers studied peristaltic transport of non-Newtonian fluids [11].RaghunathaRaoand Prasad [12] have investigated the peristaltic flow of a couple stress fluids through porous medium in a channel at low Reynolds number.

The magneto hydrodynamic (MHD) flow of a fluid in a channel with contracting walls (peristaltic flow) is of interest in connection with certain problems of the movement of conductive physiological fluids such as the blood, blood pump machines and with the need for theoretical research on the operation of a peristaltic MHD compressor. Mekheimerand Al-Arabi[13] studied non-linear peristaltic transport of MHD flow through porous medium. Effects of a magnetic field on trapping through peristaltic motion for generalized Newtonian fluid in a channel has been studiedbyAbd El Hakeem et al. [14]. Peristaltically induced transport of a MHD biviscosity fluid in a non-uniform tube has been studied byEldabe et al.[15]. Effect of slip on peristaltic transport in an inclined channel 
Military Technical College

Kobry Elkobbah,

Cairo, Egypt

April 19-21,2016

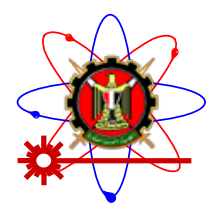

$8^{\text {th }}$ International Conference on Mathematics and Engineering Physics (ICMEP-8)

with wall effects has been considered in the work of RamanaKumariand Radhakrishnamacharya [16].Eldesoky [17]representedthe slip effects on the unsteadyMHD pulsatile blood flow through porous medium in an artery under the effectof body acceleration.El-Shehawy et al. [18] investigated the slip effects on the peristaltic flow of a non NewtonianMaxwellian fluid.Eldesoky[19] noticed the influence of slip condition on peristaltic transport of a compressible Maxwell fluidthrough porous medium in a tube. Aliet al. [20]discussed the slip effects on the peristaltic transport of MHD fluid with variable viscosity.Effects of wall properties and heat transfer on the peristaltic transport of food bolus through oesophagus was studied using a mathematical model, see Sreenadh et al. [21].

A particulate flow plays an important role in various industrial and natural processes. To predictthe influence of it in a pipeline, channel or a fitting, it is essential to characterize the effects in a simple standardized geometry.Powder technology, fluidization, sedimentation, combustion, aerosol filtration, atmospheric fallout, lunar ash flow and environmental pollution are some examples of particulate fluid suspension phenomenon. Peristaltic pumping of a particle fluid mixture has been investigated by many researchers.Medhavi and Singh [22] noted a two-layered suspension flow induced by peristaltic waves. Peristaltic motion of a particle-fluid suspension in a planarchannelhas been previously studied by Mekheimer [23]. Srivastava[24] registered the behavior of the particlefluid suspension flow induced by peristaltic waves in a circular cylindrical tube.

Because the idea is new and needs reasonable attention for the authors known, the present research is aimed to investigate the peristaltic flow of a 
Military Technical College

Kobry Elkobbah,

Cairo, Egypt

April 19-21,2016

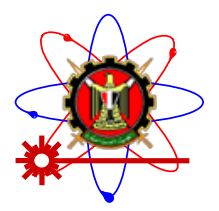

$8^{\text {th }}$ International Conference on Mathematics and Engineering

Physics (ICMEP-8)

Newtoniansuspended fluid flows through magnetic field in a flexible channel under slip condition parameter. Analytical solution using perturbation method is used to obtain the velocities, pressure gradient and streamlines. The governing equations of the fluid flow through magnetic field are solved with the presence of fluid suspensions. The behaviors of the velocity components, critical pressure, streamlines have been analyzed in either case for different sets of parameters.

\section{Mathematical Formulation}

Consider a Newtonian suspended flow of blood in an axisymmetric channel artery of mean radius dunder the effect of magnetic field. Thecartesian coordinate system $\mathrm{x}^{\prime}, \mathrm{y}^{\prime}$ are introduced, where thex'-axis lies along the center of the channel and $y^{\prime}$ is normal to it, see Fig. 1. Thewalls of the channel are assumed to be flexible with travelling sinusoidal wave on the compliant wall of channel. The wave shape is expressed as:

$$
y^{\prime}\left(x^{\prime}, t^{\prime}\right)=d+a \cos \left(\frac{2 \pi}{\lambda}\left(x^{\prime}-c_{w} t^{\prime}\right)\right)
$$

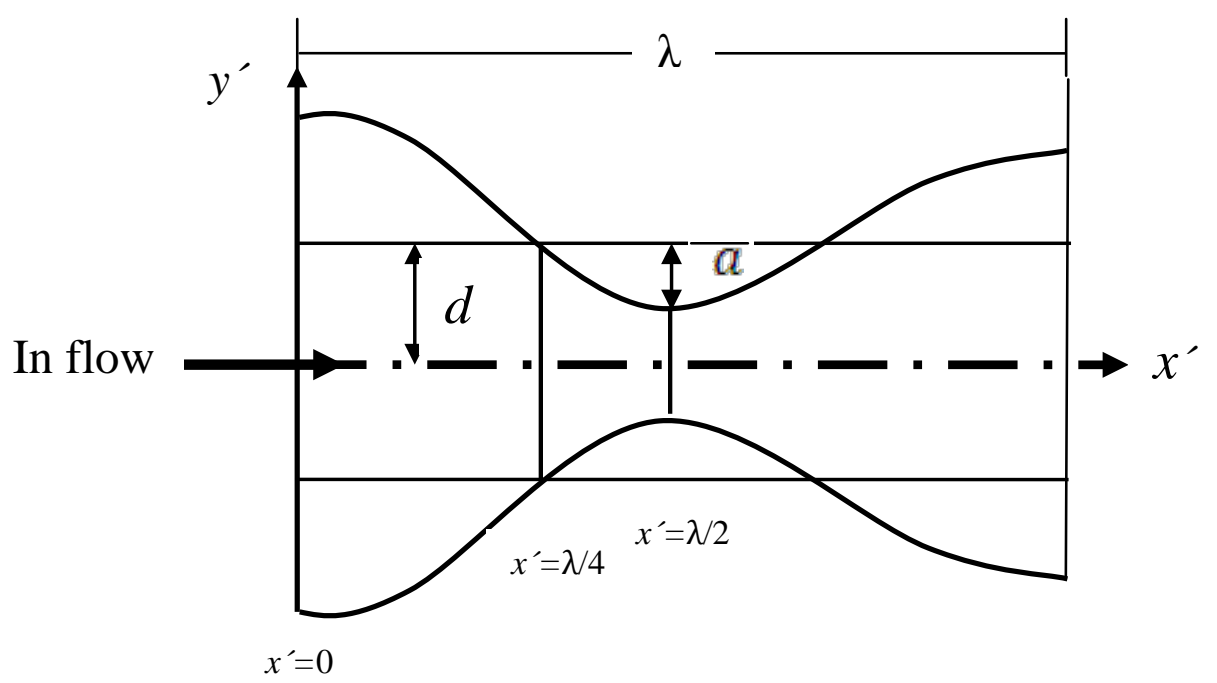

Fig. 1 Geometry of the problem 
Military Technical College

Kobry Elkobbah,

Cairo, Egypt

April 19-21,2016

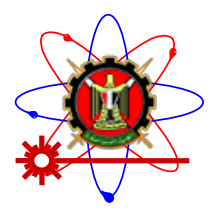

$8^{\text {th }}$ International Conference on Mathematics and Engineering

Physics (ICMEP-8)

The main governing equations are the momentum and mass for the carrying fluid and particulate (suspension) phase, respectively as follows:

For fluid phase:

(a) $\mathrm{x}^{\prime}$-momentum

$$
\begin{aligned}
& (1-C) \rho_{f}\left[\frac{\partial u_{f}^{\prime}}{\partial t^{\prime}}+u_{f}^{\prime} \frac{\partial u_{f}^{\prime}}{\partial x^{\prime}}+v_{f}^{\prime} \frac{\partial u_{f}^{\prime}}{\partial y^{\prime}}\right]=-(1-C) \frac{\partial p^{\prime}}{\partial x^{\prime}}-\sigma \beta_{o}^{2} u_{f}^{\prime} \\
& +(1-C) \mu_{s}(C)\left(\frac{\partial^{2} u_{f}^{\prime}}{\partial x^{\prime 2}}+\frac{\partial^{2} u_{f}^{\prime}}{\partial y^{\prime 2}}\right)+C S^{\prime}\left(u_{p}^{\prime}-u_{f}^{\prime}\right){ }_{(2)}
\end{aligned}
$$

(b) $\mathrm{y}^{\prime}$-momentum

$$
\begin{aligned}
& (1-C) \rho_{f}\left[\frac{\partial v_{f}^{\prime}}{\partial t^{\prime}}+u_{f}^{\prime} \frac{\partial v_{f}^{\prime}}{\partial x^{\prime}}+v_{f}^{\prime} \frac{\partial v_{f}^{\prime}}{\partial y^{\prime}}\right]=-(1-C) \frac{\partial p^{\prime}}{\partial y^{\prime}} \\
& +(1-C) \mu_{s}(C)\left(\frac{\partial^{2} v_{f}^{\prime}}{\partial x^{\prime 2}}+\frac{\partial^{2} v_{f}^{\prime}}{\partial y^{\prime 2}}\right)+C S^{\prime}\left(v_{p}^{\prime}-v_{f}^{\prime}\right),
\end{aligned}
$$

(c) conservation of mass

$$
\frac{\partial}{\partial x^{\prime}}\left((1-C) u_{f}^{\prime}\right)+\frac{\partial}{\partial y^{\prime}}\left((1-C) v_{f}^{\prime}\right)=0 \text {. }
$$

For particulate phase:

(a) $x^{\prime}$-momentum

$C \rho_{p}\left[\frac{\partial u_{p}^{\prime}}{\partial t^{\prime}}+u_{p}^{\prime} \frac{\partial u_{p}^{\prime}}{\partial x^{\prime}}+v_{p}^{\prime} \frac{\partial u_{p}^{\prime}}{\partial y^{\prime}}\right]=-C \frac{\partial p^{\prime}}{\partial x^{\prime}}+C S^{\prime}\left(u_{f}^{\prime}-u_{p}^{\prime}\right)$

(b) $\mathrm{y}^{\prime}$-momentum

$C \rho_{p}\left[\frac{\partial v_{p}^{\prime}}{\partial t^{\prime}}+u_{p}^{\prime} \frac{\partial v_{p}^{\prime}}{\partial x^{\prime}}+v_{p}^{\prime} \frac{\partial v_{p}^{\prime}}{\partial y^{\prime}}\right]=-C \frac{\partial p^{\prime}}{\partial y^{\prime}}+C S^{\prime}\left(v_{f}^{\prime}-v_{p}^{\prime}\right)$,

(c) conservation of mass

$\frac{\partial}{\partial x^{\prime}}\left(C u_{p}^{\prime}\right)+\frac{\partial}{\partial y^{\prime}}\left(C v_{p}^{\prime}\right)=0$. 


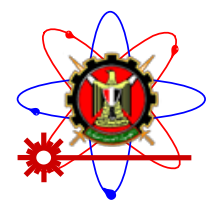

In equations from 2 to $7,\left(u_{f}{ }^{\prime}, v_{f}{ }^{\prime}\right)$ and $\left(u_{p}{ }^{\prime}, v_{p}{ }^{\prime}\right)$ represent fluid phase and particulate phase velocities in the $\mathrm{x}^{\prime}$ and $\mathrm{y}^{\prime}$ directions, respectively.

$\mathrm{C}, \mathrm{S}^{\prime}, \rho_{f}, \rho_{p}, \mu_{s}, \mathrm{p}^{\prime}, \sigma, \beta_{0}$ are particles concentration, interaction force between fluid and particle, fluid density, particle density, mixture viscosity, pressure, thermal conductivity and magnetic parameter, respectively.

The boundary conditions that must be satisfied by the fluid on the walls are the no-slip or slip conditions.

$u_{f}^{\prime}= \pm A \frac{\partial u_{f}^{\prime}}{\partial y^{\prime}}$

$u_{p}^{\prime}= \pm A \frac{\partial u_{p}^{\prime}}{\partial y^{\prime}}$

$v_{f}^{\prime}= \pm \frac{\partial \eta}{\partial t^{\prime}}$

$v_{p}^{\prime}= \pm \frac{\partial \eta}{\partial t^{\prime}}$

at the wall defined by equation (1).

The stream function relations with velocity are defined as:

$u_{f}^{\prime}=\frac{\partial \psi_{f}^{\prime}}{\partial y^{\prime}}, v_{f}^{\prime}=-\frac{\partial \psi_{f}^{\prime}}{\partial x^{\prime}}, u_{p}^{\prime}=\frac{\partial \psi_{p}^{\prime}}{\partial y^{\prime}}, v_{p}^{\prime}=-\frac{\partial \psi_{p}^{\prime}}{\partial x^{\prime}}$,

and can be used in equations 2-11 to yield the followings:

$$
\begin{array}{r}
(1-C) \rho_{f}\left[\frac{\partial}{\partial t^{\prime}} \nabla^{2} \psi_{f}^{\prime}+\psi_{f_{y}}^{\prime} \nabla^{2} \psi_{f_{x}}^{\prime}-\psi_{f_{x}}^{\prime} \nabla^{2} \psi_{f_{y}}^{\prime}\right]=-\sigma \beta_{o}^{2} \psi_{f_{y y}}^{\prime}+ \\
(1-C) \mu_{s}(C) \nabla^{4} \psi_{f}^{\prime}+C S^{\prime}\left(\nabla^{2} \psi_{p}^{\prime}-\nabla^{2} \psi_{f}^{\prime}\right) \\
C \rho_{p}\left[\frac{\partial}{\partial t^{\prime}} \nabla^{2} \psi_{p}^{\prime}+\psi_{p_{y}}^{\prime} \nabla^{2} \psi_{p_{x}}^{\prime}-\psi_{p_{x}}^{\prime} \nabla^{2} \psi_{p_{y}}^{\prime}\right]=C S^{\prime}\left(\nabla^{2} \psi_{f}^{\prime}-\nabla^{2} \psi_{p}^{\prime}\right)
\end{array}
$$




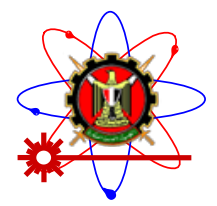

The wall boundary conditions based on the stream function forms can be also rewritten as:

$$
\begin{aligned}
& \psi_{f_{y}}^{\prime}= \pm A \frac{\partial \psi_{f_{y}}^{\prime}}{\partial y^{\prime}} \\
& \psi_{p_{y}}^{\prime}= \pm A \frac{\partial \psi_{p_{y}}^{\prime}}{\partial y^{\prime}} \\
& \psi_{f_{x}}^{\prime}= \pm \frac{2 \pi a c_{w}}{\lambda} \sin \frac{2 \pi}{\lambda}\left(x^{\prime}-c_{w} t^{\prime}\right) \\
& \psi_{p_{x}}^{\prime}= \pm \frac{2 \pi a c_{w}}{\lambda} \sin \frac{2 \pi}{\lambda}\left(x^{\prime}-c_{w} t^{\prime}\right)
\end{aligned}
$$

where A is the mean free path.

In real characteristic of peristalsis of the ureter, one end of which is joined to kidney at the pelvis and the other end is joined to the bladder, the end conditions are hydroelastic in nature. The pressure gradient depends on the amplitude of the wave motion and can be written as:

$$
\left(\frac{\partial p^{\prime}}{\partial x^{\prime}}\right)=\left(\frac{\partial p^{\prime}}{\partial x^{\prime}}\right)_{0}+a\left(\frac{\partial p^{\prime}}{\partial x^{\prime}}\right)_{1}+a^{2}\left(\frac{\partial p^{\prime}}{\partial x^{\prime}}\right)_{2} \cdot \text { Introducing the following dimensionless }
$$

variables $y=\frac{y^{\prime}}{d}, x=\frac{x^{\prime}}{d}, \eta=\frac{\eta^{\prime}}{d}, u_{f}=\frac{u_{f}^{\prime}}{c_{w}}, v_{f}=\frac{v_{f}^{\prime}}{c_{w}}, u_{p}=\frac{u_{p}^{\prime}}{c_{w}}, v_{p}=\frac{v_{p}^{\prime}}{c_{w}}$,

$$
\psi_{f}=\frac{\psi_{f}^{\prime}}{c_{w} d}, \psi_{p}=\frac{\psi_{p}^{\prime}}{c_{w} d}, S=\frac{S^{\prime} a^{2}}{\mu_{o}}, \mu=\frac{\mu_{s}}{\mu_{o}}, t=\frac{c_{w} t^{\prime}}{d}, p=\frac{p^{\prime}}{\rho_{f} c_{w}^{2}} \text {, and consideringthe }
$$

non-dimensional following parameters:

- $\quad$ Knudsen number $K n=\frac{A}{d}$ 
Military Technical College

Kobry Elkobbah,

Cairo, Egypt

April 19-21,2016

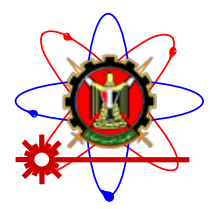

$8^{\text {th }}$ International Conference on Mathematics and Engineering

Physics (ICMEP-8)

- $\quad$ Suspension Reynolds number $\operatorname{Re}=\frac{c_{w} d \rho_{f}}{(1-C) \mu_{s}}$

- Suspension parameters $M=\frac{S^{\prime} d^{2}}{(1-C) \mu_{s}}, N=\frac{S^{\prime} d^{2} \rho_{f}}{(1-C) \mu_{s} \rho_{p}}$

- $\quad$ Magnetic parameter $M m=\frac{\sigma \beta_{o}^{2} d}{c_{w} \rho_{f}}$

- Wave number $\alpha=\frac{2 \pi d}{\lambda}$

- Amplitude ratio $\varepsilon=\frac{a}{d}$

Introducing the previous non-dimensional parameters, the equations of the fluid and the particle can be written as:

$$
\begin{aligned}
& (1-C) \operatorname{Re}\left[\frac{\partial}{\partial t} \nabla^{2} \psi_{f}+\psi_{f_{y}} \nabla^{2} \psi_{f_{x}}-\psi_{f_{x}} \nabla^{2} \psi_{f_{y}}\right]=\nabla^{4} \psi_{f}+C M\left(\nabla^{2} \psi_{f}-\nabla^{2} \psi_{p}\right)-\gamma^{2} \psi_{f_{y y}} \\
& C \operatorname{Re}\left[\frac{\partial}{\partial t} \nabla^{2} \psi_{p}+\psi_{p_{y}} \nabla^{2} \psi_{p_{x}}-\psi_{p_{x}} \nabla^{2} \psi_{p_{y}}\right]=C N\left(\nabla^{2} \psi_{f}-\nabla^{2} \psi_{p}\right) \\
& \eta(x, t)=\varepsilon \cos \alpha(x-t) \\
& \text { and } \gamma^{2}=\operatorname{ReMm} \text {. }
\end{aligned}
$$

The non-dimensional boundary conditions are:

$\psi_{f_{y}}= \pm K n \frac{\partial \psi_{f_{y}}}{\partial y}$,
$\psi_{p_{y}}= \pm K n \frac{\partial \psi_{p_{y}}}{\partial y}$,

$\psi_{f_{x}}= \pm \varepsilon \alpha \sin \alpha(x-t)$

(22) $\psi_{p_{x}}= \pm \varepsilon \alpha \sin \alpha(x-t)$, 
Military Technical College

Kobry Elkobbah,

Cairo, Egypt

April 19-21,2016 $8^{\text {th }}$ International Conference on Mathematics and Engineering

Physics (ICMEP-8)

at the wall, that can be non-dimensionlized to be:

$y= \pm 1 \pm \eta(x, t)$.

The pressure gradient equation is now non-dimensionlized to be:

$\left(\frac{\partial p}{\partial x}\right)=\left(\frac{\partial p}{\partial x}\right)_{0}+\varepsilon\left(\frac{\partial p}{\partial x}\right)_{1}+\varepsilon^{2}\left(\frac{\partial p}{\partial x}\right)_{2}$

\section{Solution method:}

By assuming the amplitude ratio $(\varepsilon)$ of the wave to be small, the solution of the

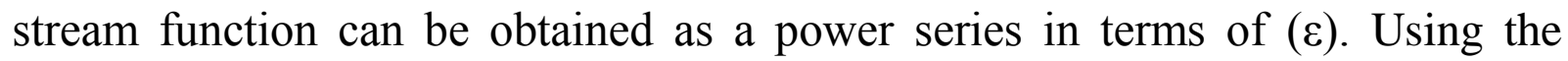
perturbation method, the stream function can be written as:

$$
\begin{aligned}
& \psi_{f}=\psi_{f_{0}}+\varepsilon \psi_{f_{1}}+\varepsilon^{2} \psi_{f_{2}}+\ldots \ldots \ldots . . \\
& \psi_{p}=\psi_{p_{0}}+\varepsilon \psi_{p_{1}}+\varepsilon^{2} \psi_{p_{2}}+\ldots \ldots \ldots . .
\end{aligned}
$$

Substituting the perturbation parameter into the equations 18 to 23 .

By comparing the coefficients of $\left(\varepsilon^{0}, \varepsilon^{1}, \varepsilon^{2}\right)$ in the equations 18 and 19 , the following coefficients can be formulated:

Coefficient of $\left(\varepsilon^{0}\right)$ :

$(1-C) \operatorname{Re}\left[\frac{\partial}{\partial t} \nabla^{2} \psi_{f_{0}}+\psi_{f_{0_{y}}} \nabla^{2} \psi_{f_{0_{x}}}-\psi_{f_{0_{x}}} \nabla^{2} \psi_{f_{0_{y}}}\right]=\nabla^{4} \psi_{f_{0}}+C M\left(\nabla^{2} \psi_{p_{0}}-\nabla^{2} \psi_{f_{0}}\right)-\gamma^{2} \psi_{f_{0 y y}}($

$C \operatorname{Re}\left[\frac{\partial}{\partial t} \nabla^{2} \psi_{p_{0}}+\psi_{p_{0_{y}}} \nabla^{2} \psi_{p_{0_{x}}}-\psi_{p_{0_{x}}} \nabla^{2} \psi_{p_{0_{y}}}\right]=C N\left(\nabla^{2} \psi_{f_{0}}-\nabla^{2} \psi_{p_{0}}\right)$

Coefficient of $\left(\varepsilon^{1}\right)$ :

$(1-C) \operatorname{Re}\left[\frac{\partial}{\partial t} \nabla^{2} \psi_{f_{1}}+\psi_{f_{0 y}} \nabla^{2} \psi_{f_{1 x}}+\psi_{f_{1 y}} \nabla^{2} \psi_{f_{0_{x}}}-\psi_{f_{0_{x}}} \nabla^{2} \psi_{f_{1 y}}-\psi_{f_{1 x}} \nabla^{2} \psi_{f_{0 y}}\right]=\nabla^{4} \psi_{f_{1}}+C M\left(\nabla^{2} \psi_{p_{1}}-\nabla^{2} \psi_{f_{1}}\right)-\gamma^{2} \psi_{f_{1 y y}}(2$

8) 
Military Technical College Kobry Elkobbah,

Cairo, Egypt

April 19-21,2016 $8^{\text {th }}$ International Conference on Mathematics and Engineering

Physics (ICMEP-8)

$$
C \operatorname{Re}\left[\frac{\partial}{\partial t} \nabla^{2} \psi_{p_{1}}+\psi_{p_{0_{y}}} \nabla^{2} \psi_{p_{1 x}}+\psi_{p_{1 y}} \nabla^{2} \psi_{p_{0_{x}}}-\psi_{p_{0_{x}}} \nabla^{2} \psi_{p_{1 y}}-\psi_{p_{1_{x}}} \nabla^{2} \psi_{p_{0_{y}}}\right]=C N\left(\nabla^{2} \psi_{f_{1}}-\nabla^{2} \psi_{p_{1}}\right)
$$

Coefficient

of $\left(\varepsilon^{2}\right)$ :

$(1-C) \operatorname{Re}\left[\frac{\partial}{\partial t} \nabla^{2} \psi_{f_{2}}+\psi_{f_{0 y}} \nabla^{2} \psi_{f_{2 x}}+\psi_{f_{2 y}} \nabla^{2} \psi_{f_{0_{x}}}-\psi_{f_{0_{x}}} \nabla^{2} \psi_{f_{2 y}}-\psi_{f_{2 x}} \nabla^{2} \psi_{f_{0_{y}}}\right]=\nabla^{4} \psi_{f_{2}}+C M\left(\nabla^{2} \psi_{p_{2}}-\nabla^{2} \psi_{f_{2}}\right)-\gamma^{2} \psi_{f_{2 y}}$

$C \operatorname{Re}\left[\frac{\partial}{\partial t} \nabla^{2} \psi_{p_{2}}+\psi_{p_{0 y}} \nabla^{2} \psi_{p_{2 x}}+\psi_{p_{2 y}} \nabla^{2} \psi_{p_{0_{x}}}-\psi_{p_{0_{x}}} \nabla^{2} \psi_{p_{2 y}}-\psi_{p_{2 x}} \nabla^{2} \psi_{p_{0_{y}}}\right]=C N\left(\nabla^{2} \psi_{f_{2}}-\nabla^{2} \psi_{p_{2}}\right)$

The boundary conditions can then be expanded as:

$\psi_{f_{y}} \pm \eta \psi_{f_{y y}} \pm \frac{\eta^{2}}{2} \psi_{f_{y y y}} \pm \ldots \ldots \ldots . . .=0$

$\psi_{p_{y}} \pm \eta \psi_{p_{y y}} \pm \frac{\eta^{2}}{2} \psi_{p_{y y y}} \pm \ldots \ldots \ldots . . .=0$

$\psi_{f_{x}} \pm \eta \psi_{f_{x y}} \pm \frac{\eta^{2}}{2} \psi_{f_{x y y}} \pm \ldots \ldots \ldots . . .= \pm \alpha \varepsilon \sin \alpha(x-t)$

$\psi_{p_{x}} \pm \eta \psi_{p_{x y}} \pm \frac{\eta^{2}}{2} \psi_{p_{x y y}} \pm \ldots \ldots \ldots . . .= \pm \alpha \varepsilon \sin \alpha(x-t)$

Implementing the coefficients of $\left(\varepsilon^{0}, \varepsilon^{1}, \varepsilon^{2}\right)$ into the wall boundary conditions, then:

for $\left(\varepsilon^{0}\right)$

$\psi_{f_{0_{y}}}= \pm K n \psi_{f_{0_{y y}}}$

$\psi_{p_{0 y}}= \pm K n \psi_{p_{0 y y}}$

$\psi_{f_{0_{x}}}=0$

$\psi_{p_{0_{x}}}=0$ 
Military Technical College

Kobry Elkobbah,

Cairo, Egypt

April 19-21,2016 $8^{\text {th }}$ International Conference on Mathematics and Engineering

Physics (ICMEP-8)

for $\left(\varepsilon^{1}\right)$

$\psi_{f_{1 y}} \pm \psi_{f_{0_{y y}}} \cos \alpha(x-t)= \pm K n\left(\psi_{f_{1 y y}}+\psi_{f_{0_{y y y}}} \cos \alpha(x-t)\right)$

$\psi_{p_{1 y}} \pm \psi_{p_{0 y y}} \cos \alpha(x-t)= \pm K n\left(\psi_{p_{1 y y}}+\psi_{p_{0_{y y y}}} \cos \alpha(x-t)\right)$

$\psi_{f_{1_{x}}} \pm \psi_{f_{x y}} \cos \alpha(x-t)= \pm \varepsilon \sin \alpha(x-t)$

$\psi_{p_{1_{x}}} \pm \psi_{p_{0_{x y}}} \cos \alpha(x-t)= \pm \varepsilon \sin \alpha(x-t)$

for $\left(\varepsilon^{2}\right)$

$\psi_{f_{2 y}} \pm \psi_{f_{1 y y}} \cos \alpha(x-t) \pm 0.5 \psi_{f_{0 y y y}} \cos ^{2} \alpha(x-t)= \pm K n\left(\psi_{f_{2 y y}} \pm \psi_{f_{1 y y y}} \cos \alpha(x-t) \pm 0.5 \psi_{f_{0 y y y y}} \cos ^{2} \alpha(x-t)\right)$

$\psi_{p_{2 y}} \pm \psi_{p_{1 y y}} \cos \alpha(x-t) \pm 0.5 \psi_{p_{0 y y y}} \cos ^{2} \alpha(x-t)= \pm K n\left(\psi_{p_{2 y y}} \pm \psi_{p_{1 y y y}} \cos \alpha(x-t) \pm 0.5 \psi_{p_{0 y y y}} \cos ^{2} \alpha(x-t)\right)$

$$
\begin{array}{r}
\psi_{f_{2_{x}}} \pm \psi_{f_{i_{x y}}} \cos \alpha(x-t) \pm 0.5 \psi_{f_{p_{x y y}}} \cos ^{2} \alpha(x-t)=0 \\
\text { (46) } \psi_{p_{2_{x}}} \pm \psi_{p_{p_{x y}}} \cos \alpha(x-t) \pm 0.5 \psi_{p_{0_{x y y}}} \cos ^{2} \alpha(x-t)=0
\end{array}
$$

The first set of differential equations in $\psi_{(f, p)_{0}}$ subjected to the steady symmetric assumption with a constant pressure gradient in the $\mathrm{x}^{\prime}$-direction, yields the following classical Poiseuille flow for the fluid and the particulate phase, respectively:

$$
\begin{array}{r}
\psi_{f_{0}}=k\left(y-\frac{y^{3}}{3}\right) \\
\psi_{p_{0}}=k\left(y+\frac{2 y}{M}-\frac{y^{3}}{3}\right)
\end{array}
$$

In which

$k=-\frac{\operatorname{Re}}{2}\left(\frac{d p}{d x}\right)_{0}$, is the Poiseuille flow parameter. 
Military Technical College

Kobry Elkobbah,

Cairo, Egypt

April 19-21,2016 $8^{\text {th }}$ International Conference on Mathematics and Engineering

Physics (ICMEP-8)

The second and third sets of differential equations in $\psi_{(f, p)_{1}}$ and $\psi_{(f, p)_{2}}$ with theircorresponding boundary conditions are satisfied by:

$$
\begin{aligned}
& \psi_{f_{1}}=0.5\left(\phi_{f_{1}} e^{i \alpha(x-t)}+\phi_{f_{1}}^{*} e^{-i \alpha(x-t)}\right) \\
& \psi_{p_{1}}=0.5\left(\phi_{p_{1}} e^{i \alpha(x-t)}+\phi_{p_{1}}^{*} e^{-i \alpha(x-t)}\right) \\
& \psi_{f_{2}}=0.5\left(\phi_{f_{20}}+\phi_{f_{22}} e^{2 i \alpha(x-t)}+\phi_{f_{22}}^{*} e^{-2 i \alpha(x-t)}\right) \\
& \psi_{p_{2}}=0.5\left(\phi_{p_{20}}+\phi_{p_{22}} e^{2 i \alpha(x-t)}+\phi_{p_{22}} e^{-2 i \alpha(x-t)}\right)
\end{aligned}
$$

where

$$
\begin{aligned}
& \phi_{f_{1}}^{*}=\text { conjugate }\left(\phi_{f_{1}}\right) \\
& \phi_{p_{1}}^{*}=\text { conjugate }\left(\phi_{p_{1}}\right)
\end{aligned}
$$

let us consider the case in which the pressure gradient $\left(\frac{\partial p}{\partial x}\right)_{0}=0$. In this case there will be no pump if the wall wave motion stops, $k=0$. Then the equations of the fluid and the particle can be written as:

$$
\begin{aligned}
& \left(\frac{d^{2}}{d y^{2}}-\beta^{2}\right)\left(\frac{d^{2}}{d y^{2}}-\alpha^{2}\right) \phi_{f_{1}}=\gamma^{2} \frac{d^{2}}{d y^{2}} \phi_{f_{1}} \\
& \left(\frac{d^{2}}{d y^{2}}-\alpha^{2}\right) \phi_{p_{1}}=\frac{N}{N-I \alpha \operatorname{Re}} \gamma^{2}\left(\frac{d^{2}}{d y^{2}}-\alpha^{2}\right) \phi_{f_{1}}
\end{aligned}
$$

The first order wall boundary conditions:

$$
\begin{gathered}
\phi_{f_{1}}= \pm K n \phi_{f_{1 y}} \pm 1 \\
\phi_{p_{1}}= \pm K n \phi_{p_{1_{y}}} \pm 1 \\
\phi_{f_{1_{y}}}= \pm K n \phi_{f_{f_{y y}}} \\
\phi_{p_{1_{1}}}= \pm K n \phi_{p_{1 y y}}
\end{gathered}
$$


Military Technical College

Kobry Elkobbah,

Cairo, Egypt

April 19-21,2016

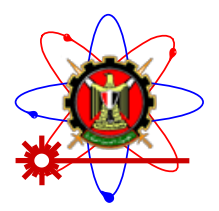

$8^{\text {th }}$ International Conference on Mathematics and Engineering

Physics (ICMEP-8)

After substituting the boundary conditions 56 and 58 into the equation 54 and the boundary conditions 57 and 59 into the equation 55, then the equations of the fluid and the particle can be written as:

$$
\begin{aligned}
& \phi_{f_{1}}=n_{1} \sinh \left(b_{1} y\right)+m_{1} \sinh \left(b_{2} y\right) \\
& \phi_{p_{1}}=n_{2} \sinh \left(b_{1} y\right)+m_{2} \sinh \left(b_{2} y\right)
\end{aligned}
$$

where

$$
\begin{aligned}
& b_{1}=\frac{1}{\sqrt{2}}\left(\alpha^{2}+\beta^{2}+\gamma^{2}+\sqrt{\left(\left(\alpha^{2}+\beta^{2}+\gamma^{2}\right)^{2}-4 \alpha^{2} \beta^{2}\right)}\right)^{0.5} \\
& b_{2}=\frac{1}{\sqrt{2}}\left(\alpha^{2}+\beta^{2}+\gamma^{2}-\sqrt{\left(\left(\alpha^{2}+\beta^{2}+\gamma^{2}\right)^{2}-4 \alpha^{2} \beta^{2}\right)}\right)^{0.5} \\
& \gamma^{2}=\operatorname{ReMm} \\
& \beta^{2}=\alpha^{2}-i \alpha \operatorname{Re}\left(1-C+\frac{C M}{N-i \alpha \operatorname{Re}}\right) \\
& n_{1}=\frac{-b_{2}\left(\cosh b_{2}-K n b_{2} \sinh b_{2}\right)}{b_{1}\left(\cosh b_{1}-K n b_{1} \sinh b_{1}\right)\left(\sinh b_{2}-K n b_{2} \cosh b_{2}\right)-b_{2}\left(\sinh b_{1}-K n b_{1} \cosh b_{1}\right)\left(\cosh b_{2}-K n b_{2} \sinh b_{2}\right)} \\
& m_{1}=\frac{b_{1}\left(\cosh b_{1}-K n b_{1} \sinh b_{1}\right)}{b_{1}\left(\cosh b_{1}-K n b_{1} \sinh b_{1}\right)\left(\sinh b_{2}-K n b_{2} \cosh b_{2}\right)-b_{2}\left(\sinh b_{1}-K n b_{1} \cosh b_{1}\right)\left(\cosh b_{2}-K n b_{2} \sinh b_{2}\right)} \\
& n_{2}=\frac{1-m_{2} \sinh \left(b_{2}\right)}{\sinh \left(b_{1}\right)} \\
& m_{2}=\frac{N m_{1}}{N-\operatorname{Re} \alpha i}
\end{aligned}
$$


Military Technical College

Kobry Elkobbah,

Cairo, Egypt

April 19-21,2016

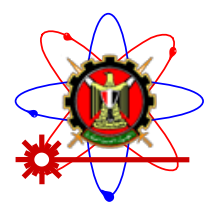

$8^{\text {th }}$ International Conference on Mathematics and Engineering

Physics (ICMEP-8)

On the other hand, substituting the boundary conditions 44 and 46 into the equation 30 and the boundary conditions 45 and 47 into the equation 31, then the second order equations of the fluid and the particle can be written as:

$$
\begin{gathered}
\phi_{f 20}^{\prime}=-\frac{i \alpha(1-C) \operatorname{Re}}{4}\left(A_{1}+A_{2}+A_{3}+A_{4}\right)-\left(-\frac{i \alpha(1-C) \operatorname{Re}}{4}\left(A_{5}+A_{6}+A_{7}+A_{8}\right)+A_{9}\right)\left(\frac{\cosh (\gamma y)}{\cosh \gamma}\right)- \\
\frac{2 C_{1}}{\gamma^{2}}\left(1-\left(\frac{\cosh (\gamma y)}{\cosh \gamma}\right)\right) \\
\phi_{p 20}^{\prime}=-\frac{i \alpha(1-C) \operatorname{Re}}{4}\left(A_{1}+A_{2}+A_{3}+A_{4}\right)+\frac{i \alpha \operatorname{Re}}{4 N}\left(B_{1}+B_{2}+B_{3}+B_{4}\right) \\
\quad-\left(-\frac{i \alpha(1-C) \operatorname{Re}}{4}\left(A_{5}+A_{6}+A_{7}+A_{8}\right)+A_{9}\right)\left(\frac{\cosh (\gamma y)}{\cosh \gamma}\right)-\frac{2 C_{1}}{\gamma^{2}}\left(1+\frac{\gamma^{2}}{M}-\left(\frac{\cosh (\gamma y)}{\cosh \gamma}\right)\right)(63) \\
\bar{u}_{f}=\frac{\varepsilon^{2}}{2}\left(\frac{i \alpha(1-C) \operatorname{Re}}{4}\left(A_{1}+A_{2}+A_{3}+A_{4}\right)-\left(-\frac{i \alpha(1-C) \operatorname{Re}}{4}\left(A_{5}+A_{6}+A_{7}+A_{8}\right)+A_{9}\right)\left(\frac{\cosh (\gamma y)}{\cosh \gamma}\right)-\right. \\
\frac{\gamma^{2}}{1}\left(1-\left(\frac{\cosh (\gamma y)}{\cosh \gamma}\right)\right)
\end{gathered}
$$

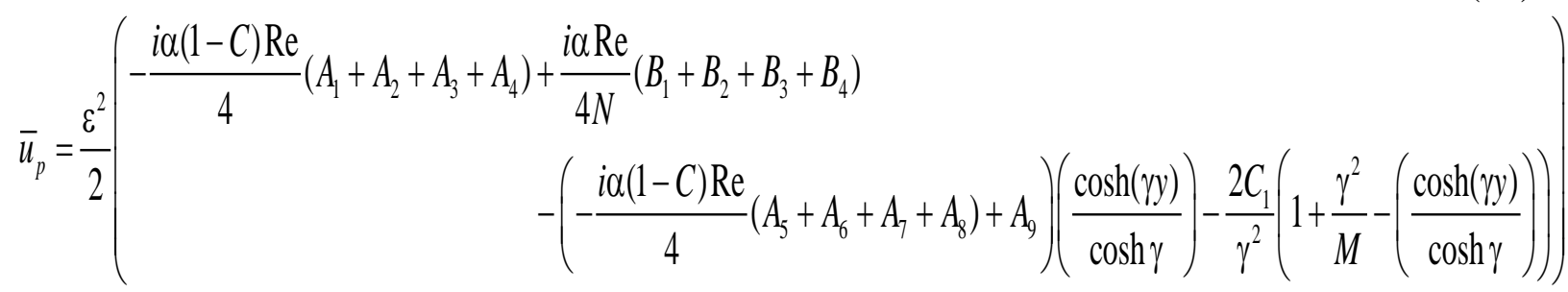

$\overline{\left(\frac{\partial p}{\partial x}\right)_{2 c . v .}}=\frac{\gamma^{2} \cosh \gamma}{2 \operatorname{Re}(\cosh \gamma-1)}\left(-\frac{i \alpha(1-C) \operatorname{Re}}{4}\left(A_{1_{0}}+A_{2_{0}}+A_{3_{0}}+A_{4_{0}}\right)-\left(-\frac{i \alpha(1-C) \operatorname{Re}}{4}\left(A_{5}+A_{6}+A_{7}+A_{8}\right)+A_{9}\right)\left(\frac{1}{\cosh \gamma}\right)\right)$ 
Military Technical College

Kobry Elkobbah,

Cairo, Egypt

April 19-21,2016

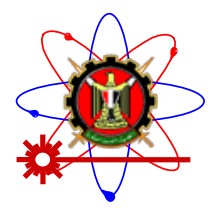

$8^{\text {th }}$ International Conference on Mathematics and Engineering

Physics (ICMEP-8)

From the equations 50 and 62, the streamlines equation can be obtained as:

$$
\psi_{f}=0.5 \varepsilon\left(\phi_{f_{1}} e^{i \alpha(x-t)}+\phi_{f_{1}}^{*} e^{-i \alpha(x-t)}\right)+0.5 \varepsilon^{2} \int_{-y}^{y} \phi_{f_{20}}^{\prime} d y
$$

where

$$
\begin{aligned}
& A_{1}=\left(b_{1}^{* 2}-b_{1}^{2}\right)\left(n_{1} n_{1}^{*}+\frac{C M}{(1-C) N} n_{2} n_{2}^{*}\right)\left(\frac{\sinh \left(\left(b_{1}+b_{1}^{*}\right) y\right)}{\left(b_{1}+b_{1}^{*}\right)\left(\left(b_{1}+b_{1}^{*}\right)^{2}-\gamma^{2}\right)}-\frac{\sinh \left(\left(b_{1}^{*}-b_{1}\right) y\right)}{\left(b_{1}^{*}-b_{1}\right)\left(\left(b_{1}^{*}-b_{1}\right)^{2}-\gamma^{2}\right)}\right) \\
& A_{2}=\left(b_{2}^{* 2}-b_{1}^{2}\right)\left(n_{1} m_{1}^{*}+\frac{C M}{(1-C) N} n_{2} m_{2}^{*}\right)\left(\frac{\sinh \left(\left(b_{1}+b_{2}^{*}\right) y\right)}{\left(b_{1}+b_{2}^{*}\right)\left(\left(b_{1}+b_{2}^{*}\right)^{2}-\gamma^{2}\right)}-\frac{\sinh \left(\left(b_{2}^{*}-b_{1}\right) y\right)}{\left(b_{2}^{*}-b_{1}\right)\left(\left(b_{2}^{*}-b_{1}\right)^{2}-\gamma^{2}\right)}\right) \\
& A_{3}=\left(b_{1}^{* 2}-b_{2}^{2}\right)\left(m_{1} n_{1}^{*}+\frac{C M}{(1-C) N} m_{2} n_{2}^{*}\right)\left(\frac{\sinh \left(\left(b_{2}+b_{1}^{*}\right) y\right)}{\left(b_{2}+b_{1}^{*}\right)\left(\left(b_{2}+b_{1}^{*}\right)^{2}-\gamma^{2}\right)}-\frac{\sinh \left(\left(b_{1}^{*}-b_{2}\right) y\right)}{\left(b_{1}^{*}-b_{2}\right)\left(\left(b_{1}^{*}-b_{2}\right)^{2}-\gamma^{2}\right)}\right) \\
& A_{4}=\left(b_{2}^{* 2}-b_{2}^{2}\right)\left(m_{1} m_{1}^{*}+\frac{C M}{(1-C) N} m_{2} m_{2}^{*}\right)\left(\frac{\sinh \left(\left(b_{2}+b_{2}^{*}\right) y\right)}{\left.\left(b_{2}+b_{2}^{*}\right)\left(b_{2}+b_{2}^{*}\right)^{2}-\gamma^{2}\right)}-\frac{\sinh \left(\left(b_{1}^{*}-b_{2}\right) y\right)}{\left(b_{2}^{*}-b_{2}\right)\left(\left(b_{2}^{*}-b_{2}\right)^{2}-\gamma^{2}\right)}\right) \\
& A_{5}=\left(b_{1}^{* 2}-b_{1}^{2}\right)\left(n_{1} n_{1}^{*}+\frac{C M}{(1-C) N} n_{2} n_{2}^{*}\right)\left(\frac{\sinh \left(b_{1}+b_{1}^{*}\right)}{\left(b_{1}+b_{1}^{*}\right)\left(\left(b_{1}+b_{1}^{*}\right)^{2}-\gamma^{2}\right)}-\frac{\sinh \left(b_{1}^{*}-b_{1}\right)}{\left(b_{1}^{*}-b_{1}\right)\left(\left(b_{1}^{*}-b_{1}\right)^{2}-\gamma^{2}\right)}\right) \\
& A_{6}=\left(b_{2}^{* 2}-b_{1}^{2}\right)\left(n_{1} m_{1}^{*}+\frac{C M}{(1-C) N} n_{2} m_{2}^{*}\right)\left(\frac{\sinh \left(b_{1}+b_{2}^{*}\right)}{\left(b_{1}+b_{2}^{*}\right)\left(\left(b_{1}+b_{2}^{*}\right)^{2}-\gamma^{2}\right)}-\frac{\sinh \left(b_{2}^{*}-b_{1}\right)}{\left(b_{2}^{*}-b_{1}\right)\left(\left(b_{2}^{*}-b_{1}\right)^{2}-\gamma^{2}\right)}\right) \\
& A_{7}=\left(b_{1}^{* 2}-b_{2}^{2}\right)\left(m_{1} n_{1}^{*}+\frac{C M}{(1-C) N} m_{2} n_{2}^{*}\right)\left(\frac{\sinh \left(b_{2}+b_{1}^{*}\right)}{\left(b_{2}+b_{1}^{*}\right)\left(\left(b_{2}+b_{1}^{*}\right)^{2}-\gamma^{2}\right)}-\frac{\sinh \left(b_{1}^{*}-b_{2}\right)}{\left(b_{1}^{*}-b_{2}\right)\left(\left(b_{1}^{*}-b_{2}\right)^{2}-\gamma^{2}\right)}\right) \\
& A_{8}=\left(b_{2}^{* 2}-b_{2}^{2}\right)\left(m_{1} m_{1}^{*}+\frac{C M}{(1-C) N} m_{2} m_{2}^{*}\right)\left(\frac{\sinh \left(b_{2}+b_{2}^{*}\right)}{\left(b_{2}+b_{2}^{*}\right)\left(\left(b_{2}+b_{2}^{*}\right)^{2}-\gamma^{2}\right)}-\frac{\sinh \left(b_{1}^{*}-b_{2}\right)}{\left(b_{2}^{*}-b_{2}\right)\left(\left(b_{2}^{*}-b_{2}\right)^{2}-\gamma^{2}\right)}\right)
\end{aligned}
$$


Military Technical College

Kobry Elkobbah,

Cairo, Egypt

April 19-21,2016 $8^{\text {th }}$ International Conference on Mathematics and Engineering Physics (ICMEP-8)

$$
\begin{aligned}
& A_{9}=0.5\left(n_{1} b_{1}^{2} \sinh \left(b_{1}\right)+m_{1} b_{2}^{2} \sinh \left(b_{2}\right)+n_{1}^{*} b_{1}^{* 2} \sinh \left(b_{1}^{*}\right)+m_{1}^{*} b_{1}^{* 2} \sinh \left(b_{1}^{*}\right)\right) \\
& B_{1}=n_{2} n_{2}^{*}\left(b_{1}^{* 2}-b_{1}^{2}\right)\left(\frac{\sinh \left(\left(b_{1}+b_{1}^{*}\right) y\right)}{\left(b_{1}+b_{1}^{*}\right)}-\frac{\sinh \left(\left(b_{1}^{*}-b_{1}\right) y\right)}{\left(b_{1}^{*}-b_{1}\right)}\right) \\
& B_{2}=n_{2} m_{2}^{*}\left(b_{2}^{* 2}-b_{1}^{2}\right)\left(\frac{\sinh \left(\left(b_{1}+b_{2}^{*}\right) y\right)}{\left(b_{1}+b_{2}^{*}\right)}-\frac{\sinh \left(\left(b_{2}^{*}-b_{1}\right) y\right)}{\left(b_{2}^{*}-b_{1}\right)}\right) \\
& B_{3}=m_{2} n_{2}^{*}\left(b_{1}^{* 2}-b_{2}^{2}\right)\left(\frac{\sinh \left(\left(b_{2}+b_{1}^{*}\right) y\right)}{\left(b_{2}+b_{1}^{*}\right)}-\frac{\sinh \left(\left(b_{1}^{*}-b_{2}\right) y\right)}{\left(b_{1}^{*}-b_{2}\right)}\right) \\
& B_{4}=m_{2} m_{2}^{*}\left(b_{2}^{* 2}-b_{2}^{2}\right)\left(\frac{\sinh \left(\left(b_{2}+b_{2}^{*}\right) y\right)}{\left(b_{2}+b_{2}^{*}\right)}-\frac{\sinh \left(\left(b_{2}^{*}-b_{2}\right) y\right)}{\left(b_{2}^{*}-b_{2}\right)}\right)
\end{aligned}
$$

In which

$b^{*}{ }_{2}=$ conjugate $\left(b_{2}\right)$

$b^{*}{ }_{1}=$ conjugate $\left(b_{1}\right)$

Equations 64, 65 (with neglecting the effect of magnetic field on the flow behavior)represent the same formula reached by Eldesoky [25].

\section{Results and discussions}

The main parameters considered in the present study are particle concentration (C), wave number $(\varepsilon)$, magnetic parameter $(\mathrm{Mm})$, slip condition $(K n)$, Reynolds number(Re) and pressure gradient $\frac{\partial p}{\partial x}$.The pressurerise is an important physical measure in the peristaltic mechanism. The chosen parameter values are $\mathrm{C}=0,0.2,0.3,0.4$ and $0.59 ; \mathrm{Mm}=0.0001,0.1,0.5$ and $1 ; \mathrm{Re}=1,10$ and $50 ; \varepsilon=0$, 0.2, 0.4 and 0.6; pressure gradient $\frac{\partial p}{\partial x}=-1,-0.3,0,0.3$ and 0.5 .The present study includes different cases for $K n=0$ (absence of slip condition) and $K n \neq 0$ (presence 
Military Technical College

Kobry Elkobbah,

Cairo, Egypt

April 19-21,2016

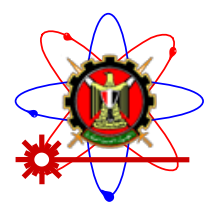

$8^{\text {th }}$ International Conference on Mathematics and Engineering Physics (ICMEP-8)

of slip condition). On the other hand, the variation of amplitude ratio $\varepsilon$ is taken into consideration. The results include the effects of different parameters on the velocity distribution, critical reflux pressure and stream lines. The velocity profiles are taken at different positions, $x^{\prime}=0, \lambda / 4$ and $\lambda / 2$.
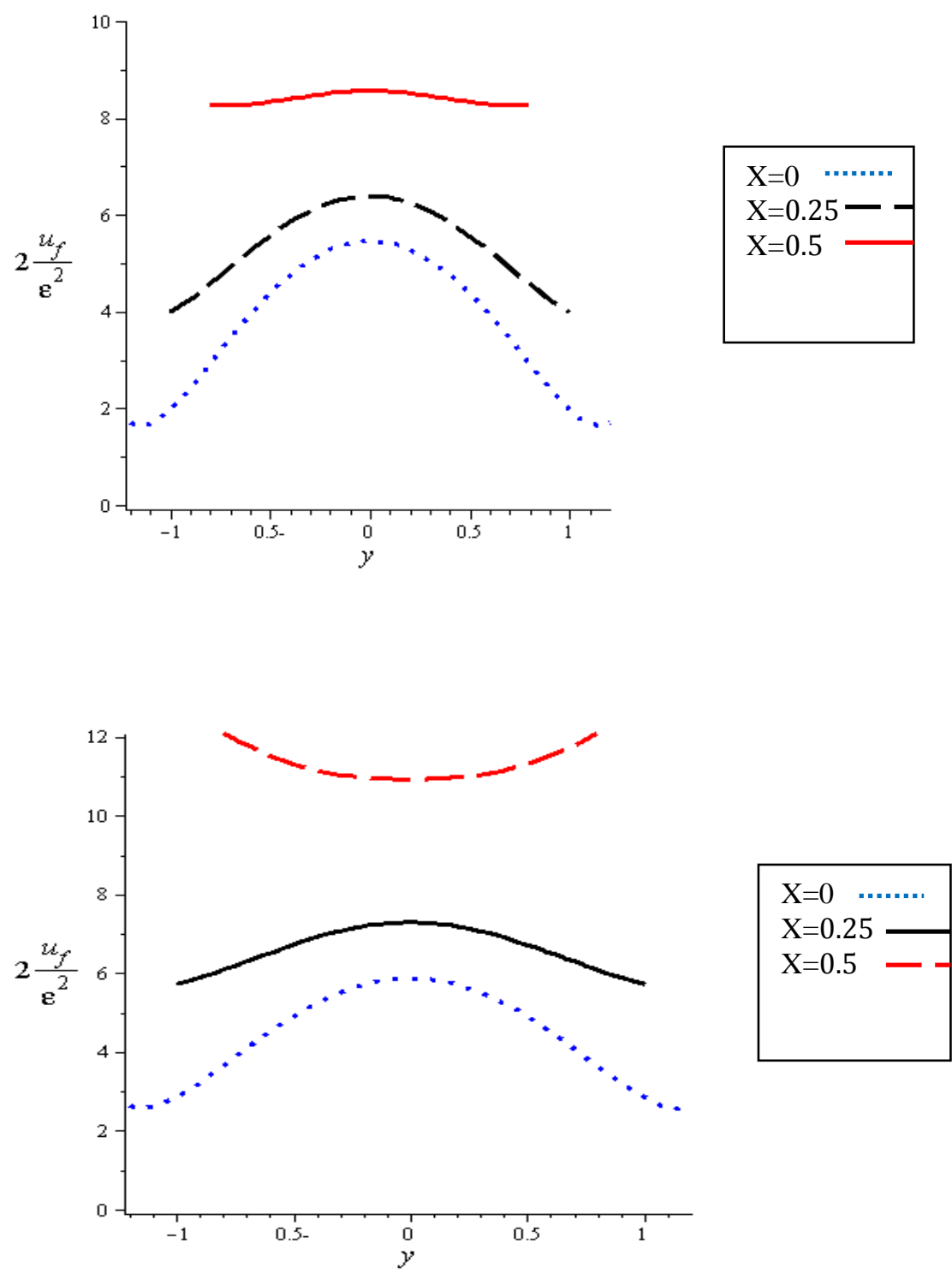

$K n=0$

$K n=0.1$

Fig. 2 Mean velocity distributionsat different locations

$$
\left(\frac{\partial p}{\partial x}=-1, \mathrm{C}=0.4, \mathrm{Mm}=0.2, \alpha=1, \operatorname{Re}=10\right)
$$


Military Technical College

Kobry Elkobbah,

Cairo, Egypt

April 19-21,2016

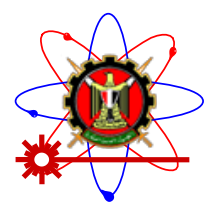

$8^{\text {th }}$ International Conference on Mathematics and Engineering Physics (ICMEP-8)

Fig. 2 shows that,the contraction of the channel enhancesthe velocity because of the conservation of mass andvice versa the velocity decreases with the expansion of the channel. The presence of slip $(K n=0.1)$ causes increase in the velocity values.
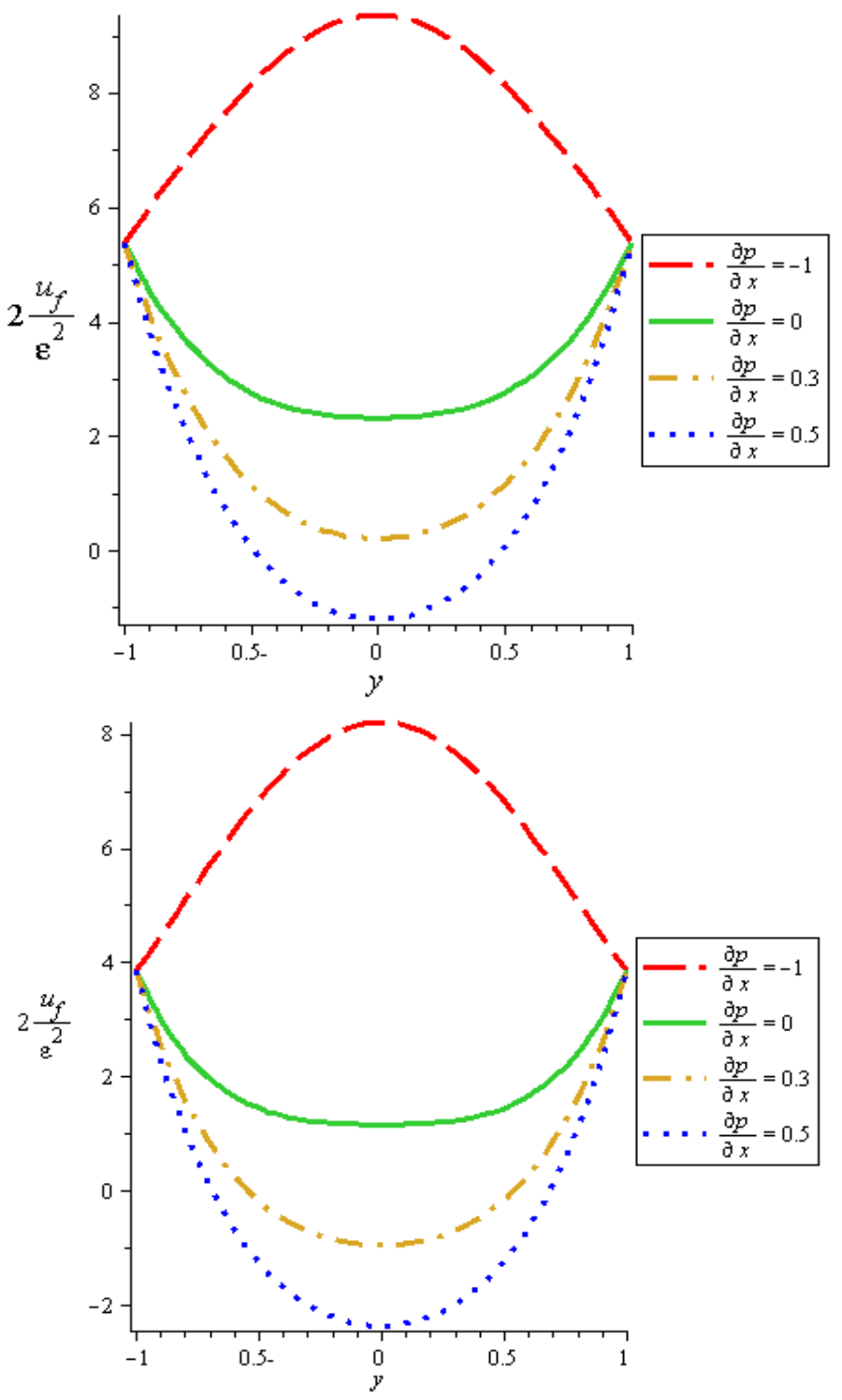

$K n=0$

$K n=0.1$

Fig. 3Mean velocity distributions with different pressure gradient $(x=0.25, \mathrm{C}=0.4, \mathrm{Mm}=0.1, \alpha=1, \mathrm{Re}=10)$ 
Military Technical College

Kobry Elkobbah,

Cairo, Egypt

April 19-21,2016

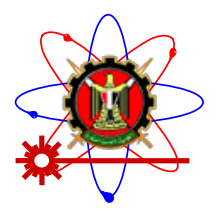

$8^{\text {th }}$ International Conference on Mathematics and Engineering Physics (ICMEP-8)

The reversal flow appears withthe adverse pressure gradient $\left(\frac{\partial p}{\partial x}>0\right)$, with delaying of back flow in the presence of slip. The approached velocity profile takes the known parabolic form of laminar flow $\left(\frac{\partial p}{\partial x}<0\right)$, while with adverse pressure gradient, back flow velocity profiles appearwith increasing back intensity at higher adverse pressure gradient. The slip condition causes the decreasing of the reversal flow, see Fig. 3.

The presence of particle concentration strongly affects the velocity. For both slip and no-slip conditions, the reversal flow increases with increasing suspension concentration (C), see Fig. 4. This appears in our life such as urine in which solute particles are suspended (urine of a diseased kidney) is more susceptible to reversal flow in ureter, in comparison to pure urine without solute particles. However, comparing the present case (very low magnetic field effect) and that for case of high magnetic field (see Fig. 5), the magnetic field increases the back flow intensity. 
Military Technical College Kobry Elkobbah,

Cairo, Egypt

April 19-21,2016

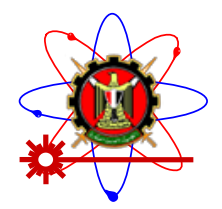

$8^{\text {th }}$ International Conference on Mathematics and Engineering

Physics (ICMEP-8)
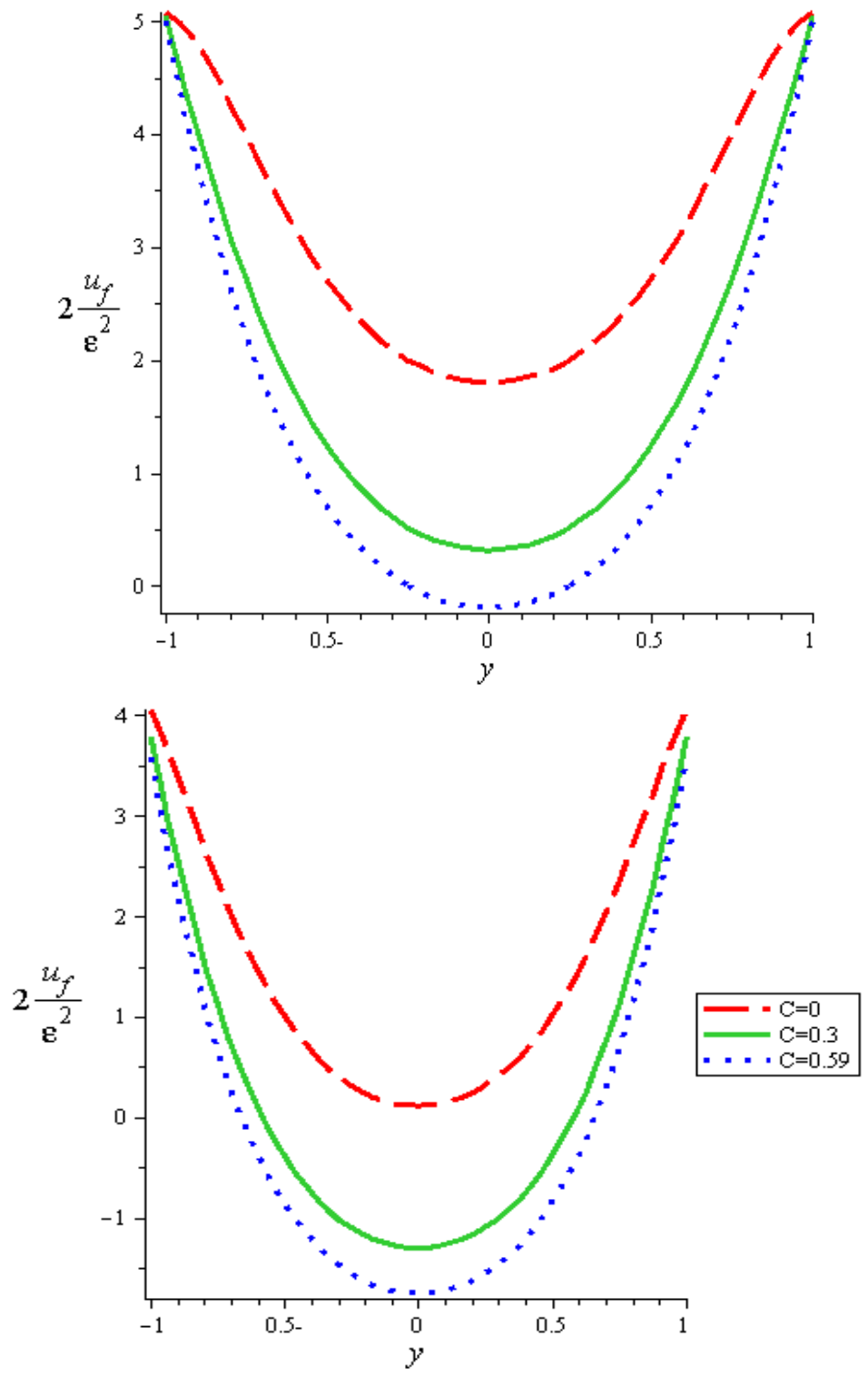

$K n=0$

$K n=0.1$

Fig. 4 Mean velocity distributions with particle concentration $\left(\frac{\partial p}{\partial x}=0.3, x=0.25, \operatorname{Mm}=0.00001, \alpha=1, \operatorname{Re}=10\right)$ 
Military Technical College Kobry Elkobbah,

Cairo, Egypt

April 19-21,2016 $8^{\text {th }}$ International Conference on Mathematics and Engineering Physics (ICMEP-8)
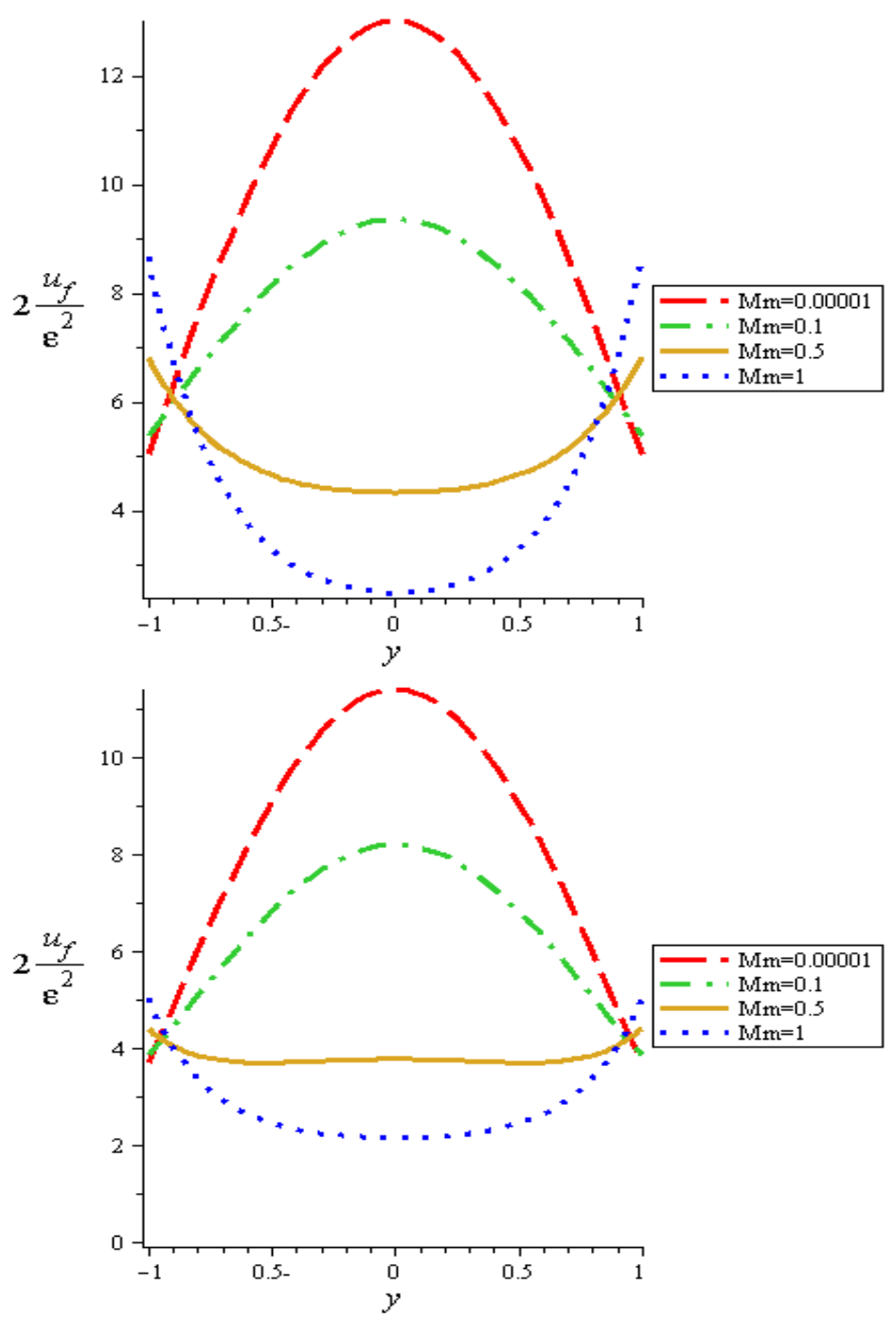

$K n=0$

$K n=0.1$

Fig. 5Mean velocity distribution with magnetic field

$$
\left(\frac{\partial p}{\partial x}=-1, \mathrm{C}=0.4, \mathrm{Re}=10, \alpha=1, x=0.25\right)
$$


Military Technical College

Kobry Elkobbah,

Cairo, Egypt

April 19-21,2016

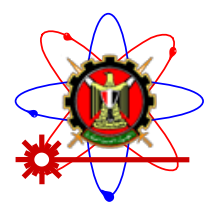

$8^{\text {th }}$ International Conference on Mathematics and Engineering Physics (ICMEP-8)
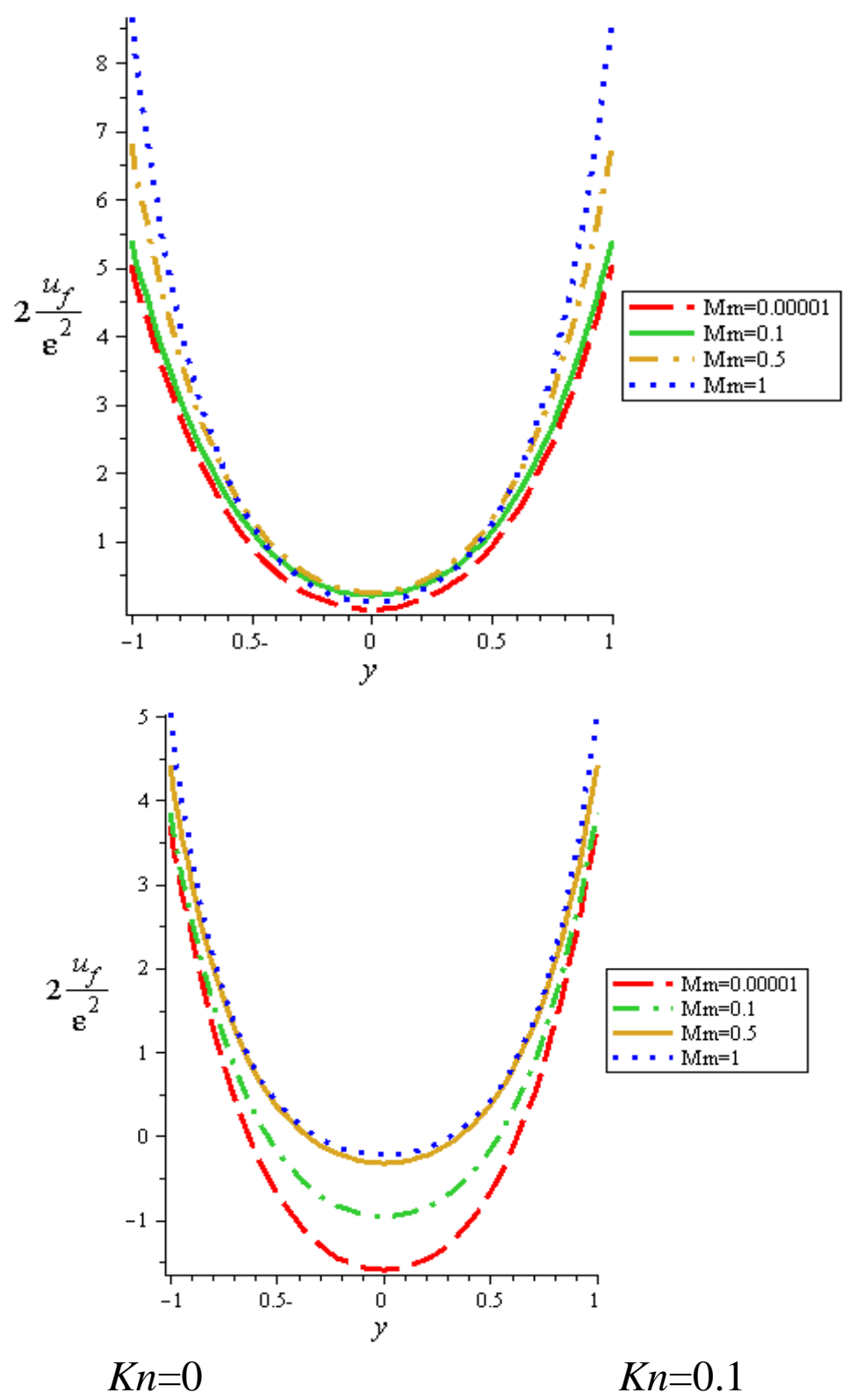

Fig. 6 Mean velocity distribution with magnetic field

$$
\left(\frac{\partial p}{\partial x}=0.3, \mathrm{C}=0.4, \operatorname{Re}=10, \alpha=1, x=0.25\right)
$$


Military Technical College Kobry Elkobbah,

Cairo, Egypt

April 19-21,2016 $8^{\text {th }}$ International Conference on Mathematics and Engineering Physics (ICMEP-8)
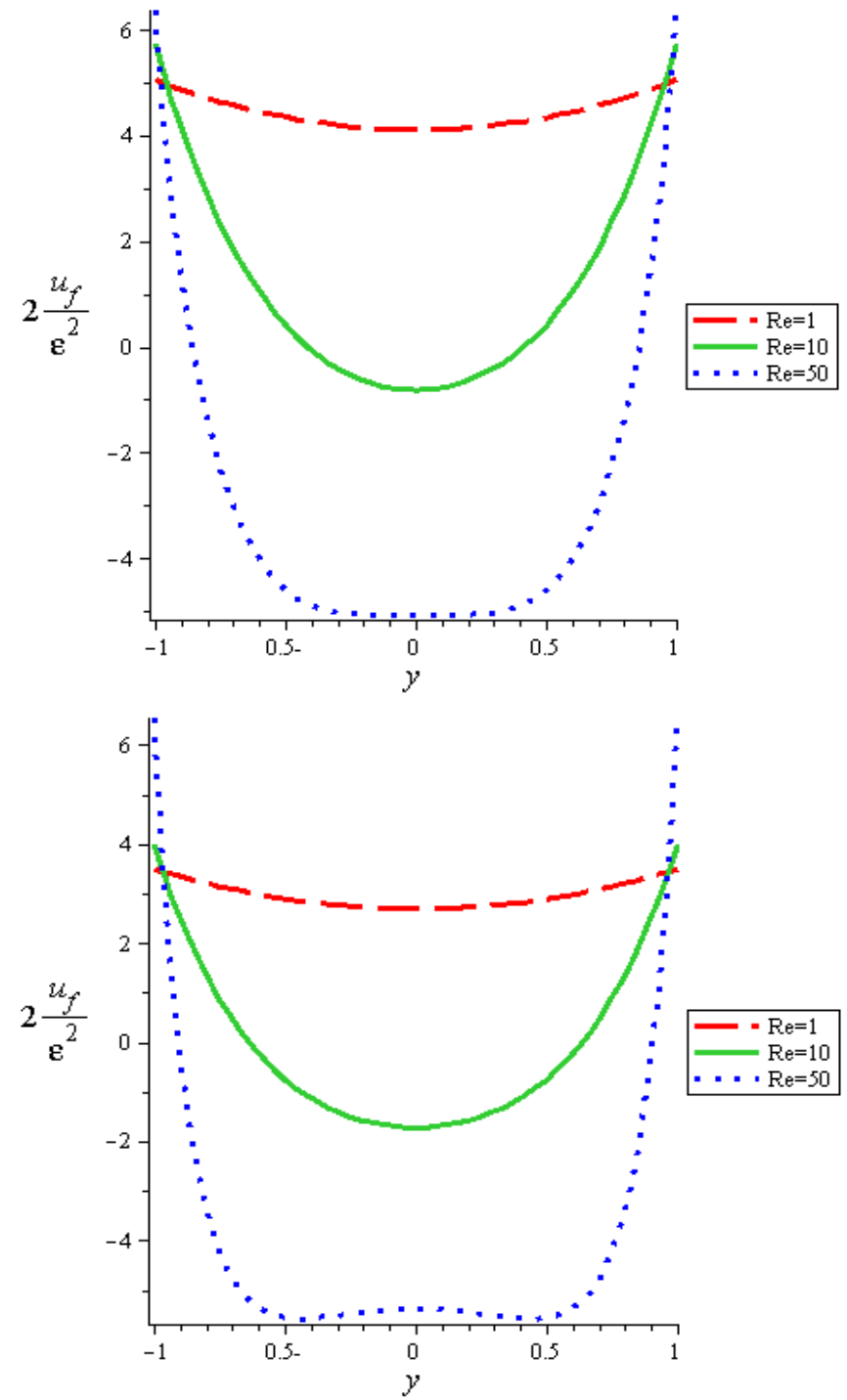

$K n=0$

$K n=0.1$

Fig. 7Mean velocity distributions with Reynolds number $\left(\frac{\partial p}{\partial x}=0.5, \mathrm{C}=0.4, \mathrm{Mm}=0.2, \alpha=1, x=0.25\right)$ 
Military Technical College

Kobry Elkobbah,

Cairo, Egypt

April 19-21,2016

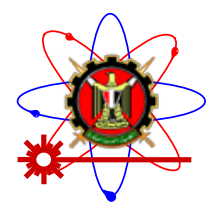

$8^{\text {th }}$ International Conference on Mathematics and Engineering Physics (ICMEP-8)

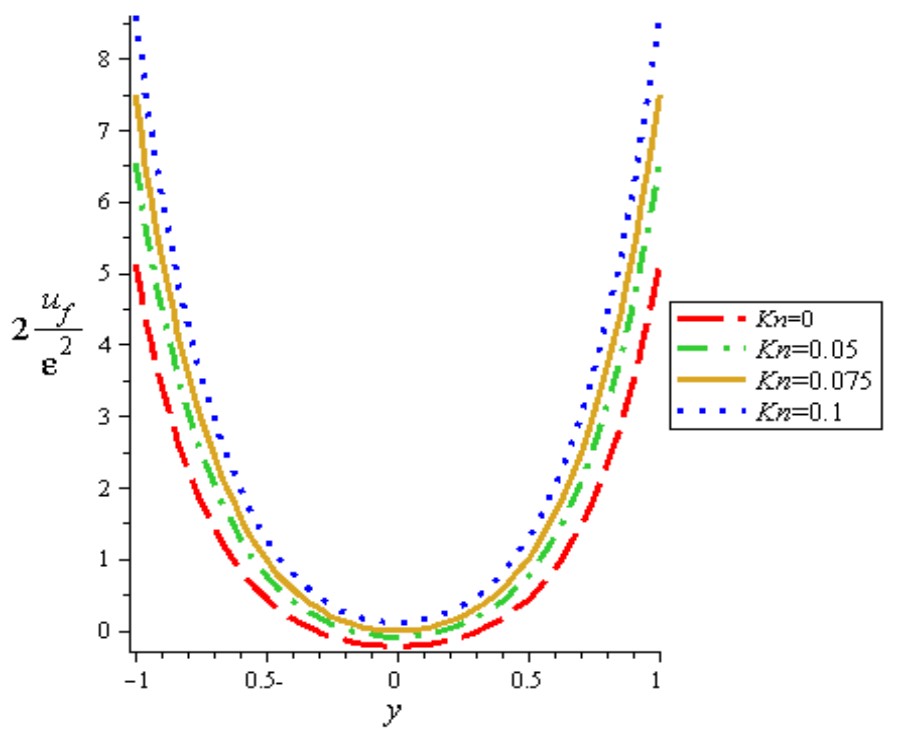

Fig. 8 Mean velocity distributions with Knudsen number

$$
\left(\frac{\partial p}{\partial x}=0.3, \mathrm{C}=0.2, \mathrm{Mm}=1, \alpha=1, \operatorname{Re}=10\right)
$$

The velocity decreases with the magnetic field awayfrom the wall, while the velocity increases with the magnetic field approaching the wallat negative pressure gradient, see Fig. 5. Fig. 6 shows that withadverse pressure gradient $\left(\frac{\partial p}{\partial x}>0\right)$ and without slip condition, the reversal velocity decreases with increasing the magnetic field. However, the presence of slip condition causes a little effect of magnetic field on the velocity distribution a way of the wall. The influence of Reynolds number on the mean velocity distribution is investigated in Fig.7. For both slip condition and no-slip condition the velocity profiles are approached to each other at high Reynolds number. However, the difference between slip condition and no slip condition appears at low Reynolds number.Fig. 8 represents the effect of Knudsen number on the mean velocity distribution. The presence of the slip condition causes increasing in the mean velocity. 
Military Technical College Kobry Elkobbah,

Cairo, Egypt

April 19-21,2016 $8^{\text {th }}$ International Conference on Mathematics and Engineering Physics (ICMEP-8)
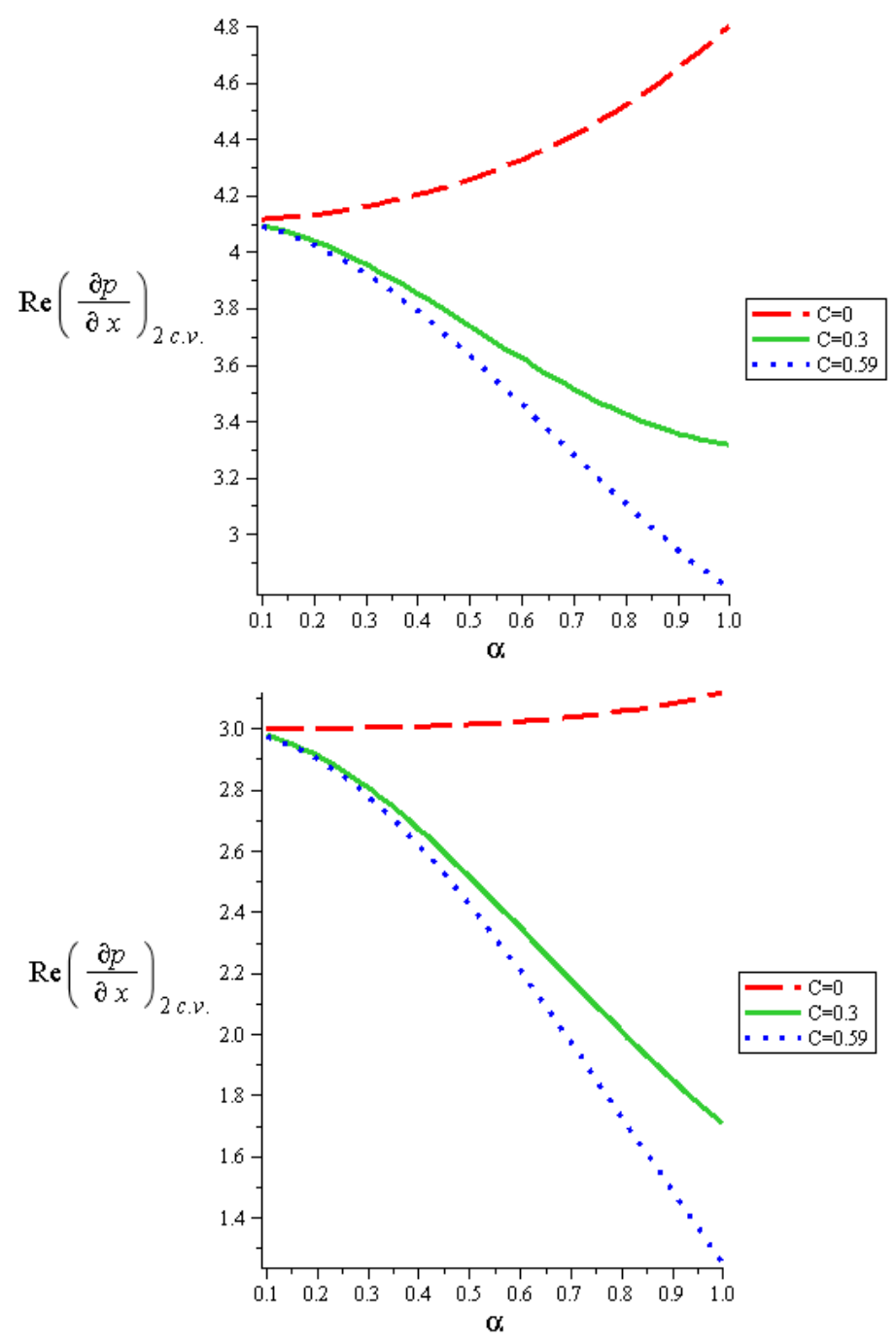

$K n=0$

$K n=0.1$

Fig. 9 Critical reflux pressure gradient with wave number at different values of $\mathrm{C}$ $(\operatorname{Re}=10, M m=0.00001)$. 
Military Technical College Kobry Elkobbah,

Cairo, Egypt

April 19-21,2016 $8^{\text {th }}$ International Conference on Mathematics and Engineering Physics (ICMEP-8)
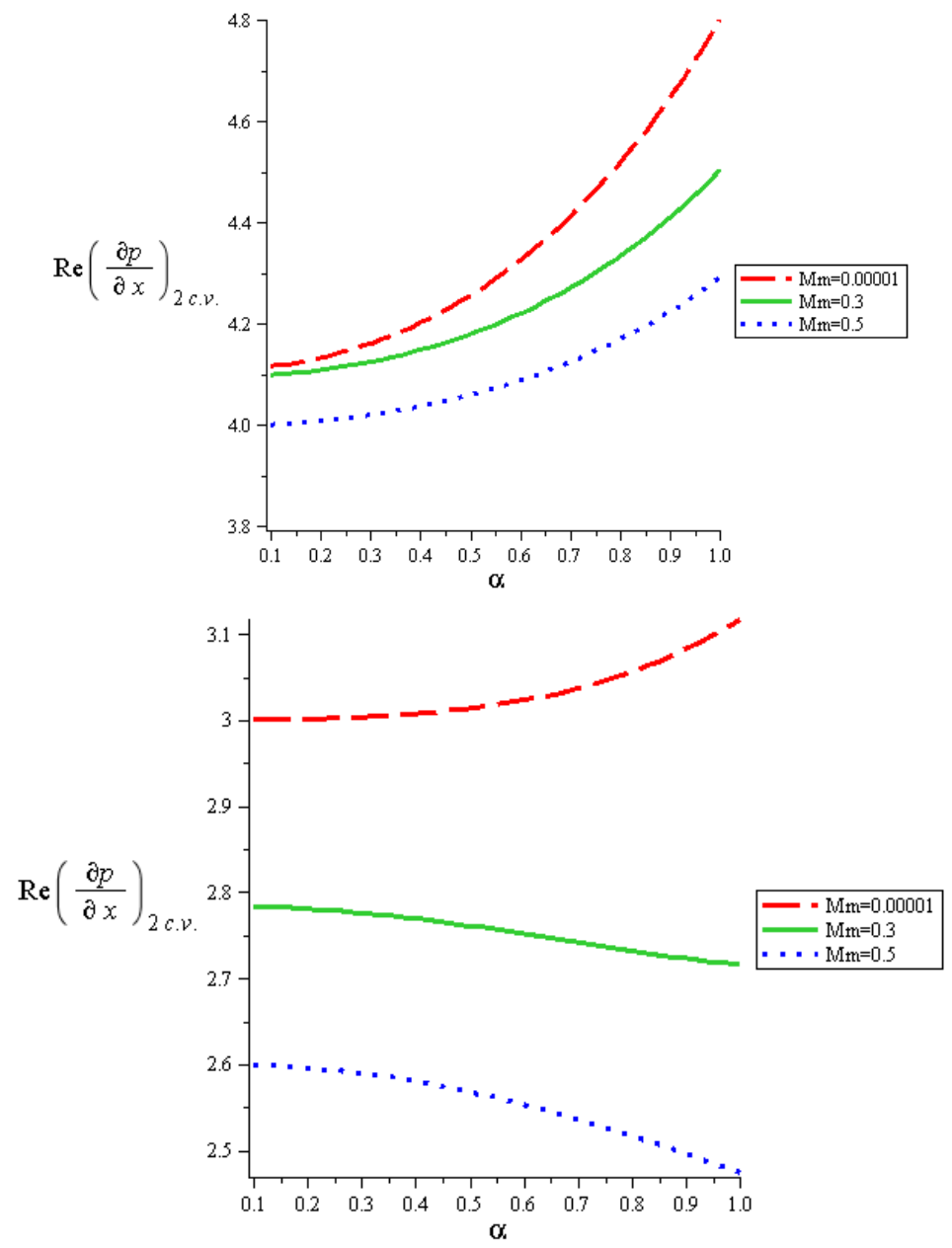

$K n=0$

$K n=0.1$

Fig. 10 Critical reflux pressure gradient with wave number at different values of $\operatorname{Mm}(\mathrm{Re}=10, \mathrm{C}=0)$. 
Military Technical College

Kobry Elkobbah,

Cairo, Egypt

April 19-21,2016 $8^{\text {th }}$ International Conference on Mathematics and Engineering Physics (ICMEP-8)

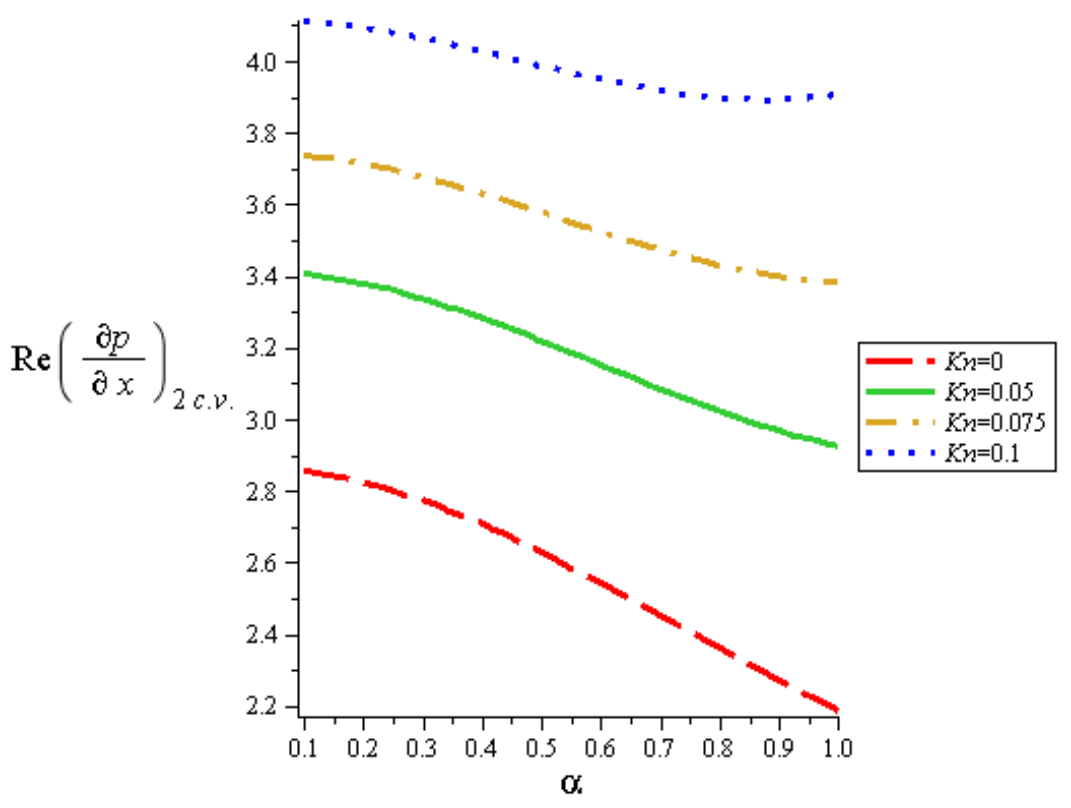

Fig. 11Critical reflux pressure with Knudsen number $(\mathrm{Mm}=0.2, \mathrm{C}=0.2, \alpha=1$, $\mathrm{Re}=10)$.

For both slip condition and no-slip condition, the values of critical reflux pressure for different values of C, Mm,Kn and $\alpha$ are shown in Figs. 9, 10 and 11. Fig. 9 represents the values of critical reflux pressure with the wave number for different values of C. The critical reflux pressure decreases with increasing the particle concentration. However, the fluid suspension has significant influence on the critical reflux pressure at $\theta \sigma \leq 1$.At the absence of fluid suspension $(\mathrm{C}=0)$, the variation of the critical reflux pressure at slip condition is higher than at no-slip condition. On the other hand,Fig. 9 shows generally that the presence of slip increases the critical reflux pressure and the presence of particle concentration causes flow reversal. The effect of magnetic field is shown in Fig. 10. The variationof the critical reflux pressure at no-slip condition is higher than that at slip condition for different values of magnetic field. At no-slip condition, the critical reflux pressure decreases with increasing the wave number at 
Military Technical College

Kobry Elkobbah,

Cairo, Egypt

April 19-21,2016

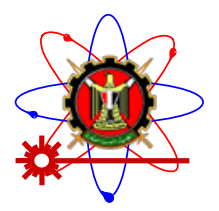

$8^{\text {th }}$ International Conference on Mathematics and Engineering Physics (ICMEP-8)

highervalues of the magnetic field, but approaching the case of ( $\mathrm{Mm}=0.00001)$, the critical reflux pressure increases with increasing the wave number. However, with slip condition, the critical reflux pressure increases with increasing the wave number at all values of the magnetic field. The effect of Knudsen number on the critical reflux pressure is analyzed in Fig. 11, where the critical reflux pressure decreases with increasing the wave number at different values of the Knudsen number.
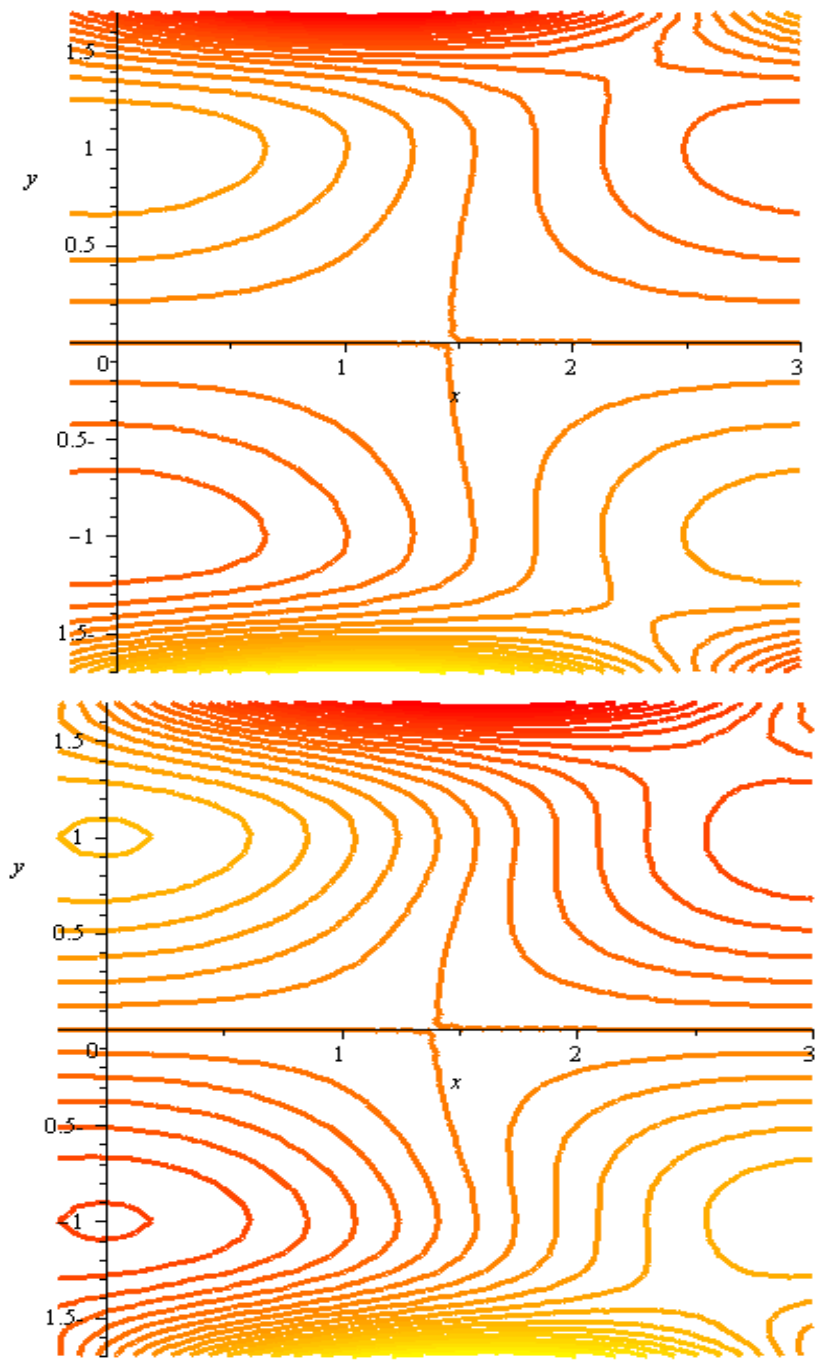

$\mathrm{Mm}=0$

$\mathrm{Mm}=1$

Fig. 12 Streamlines at different values of Mm 
Military Technical College Kobry Elkobbah,

Cairo, Egypt

April 19-21,2016

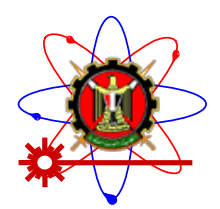

$8^{\text {th }}$ International Conference on Mathematics and Engineering Physics (ICMEP-8)
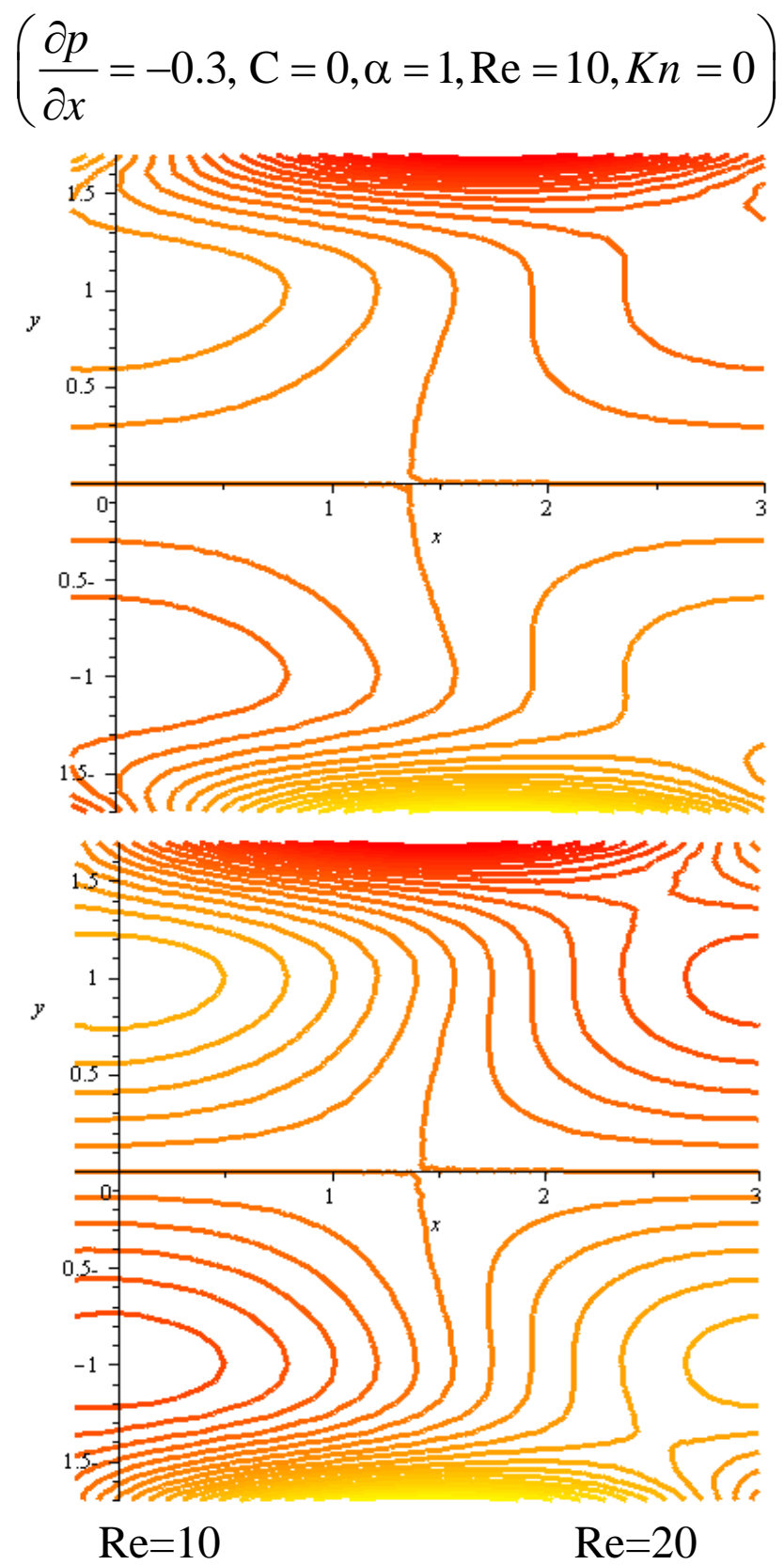

Fig. 13Streamlines at different values of Re $\left(\frac{\partial p}{\partial x}=-0.3, \mathrm{C}=0, \mathrm{Mm}=0.2, \alpha=1, K n=0\right)$ 
Military Technical College Kobry Elkobbah,

Cairo, Egypt

April 19-21,2016

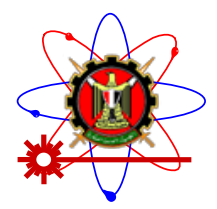

$8^{\text {th }}$ International Conference on Mathematics and Engineering Physics (ICMEP-8)
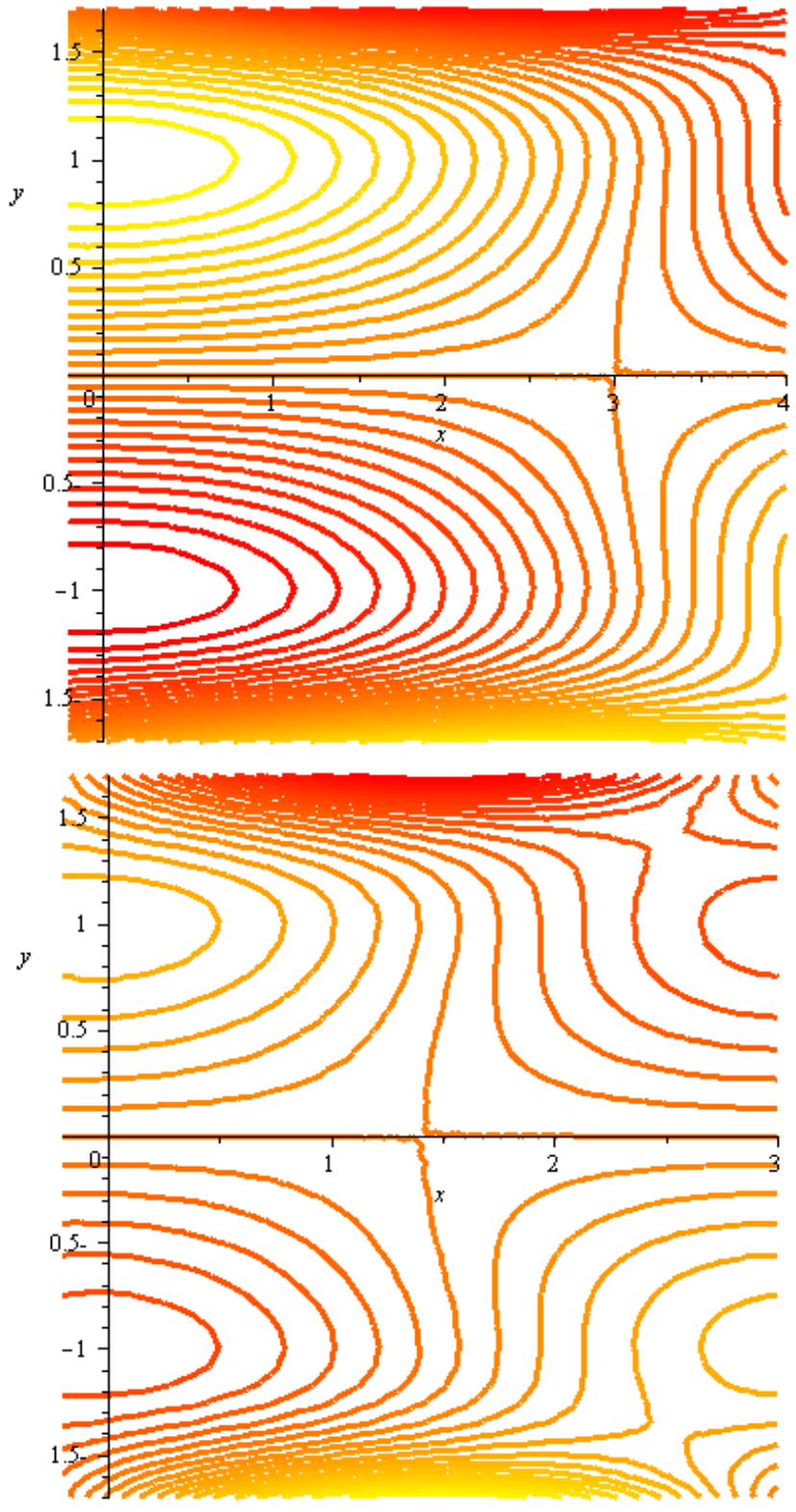

$\alpha=1$

$\alpha=0.5$

Fig. 14Streamlines at different values of $\alpha$

$\left(\frac{\partial p}{\partial x}=-0.3, \mathrm{C}=0, \mathrm{Mm}=0.2, \operatorname{Re}=10, K n=0\right)$

The effect of magnetic field on the streamlines is illustrated in Fig. 12. As noticed, the streamlines without magnetic field are crowded than that with magnetic field. The streamlines at different values of Reynolds number are 
Military Technical College

Kobry Elkobbah,

Cairo, Egypt

April 19-21,2016

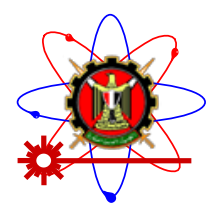

$8^{\text {th }}$ International Conference on Mathematics and Engineering Physics (ICMEP-8)

shown in Fig. 13. It can be concluded that increasing Reynolds number causes increasing the velocity of the flow. The influence of the wave number on the streamlines is analyzed in Fig. 14,in which the streamlines approach to each other with decreasing the wave number.

\section{Conclusions}

This study represents the effects of slip conditions on a particulate fluid suspension with peristaltic transport in flexible channel under the influence of MHD.The perturbation method has been used for the solution of the fluid and particle equations. The analytical solution has been developed and used for extracting velocity,critical reflux pressure and streamlines of the fluid for uniform flexible channel.The features of the flow characteristics have been analyzed using graphs and discussions. For both slipping and no slipping, main points are obtained from the present study:

- Approaching the contraction, velocity increases, while with the expansion, velocity decreases.

- For negative pressure gradient,the velocity increases, while the velocity decreases and back flow appears with adverse pressure gradient.

- The presence of particle concentration and magnetic fieldreduces the velocitywith negative pressure gradient, while the velocity increases with positive gradients.

- In the presenceof adverse pressure gradient, the Reynolds number has negative effects on the velocity values.

- The presence of slip has positive effect on the velocity.

- The critical reflux pressure gradient decreases with the presence of suspension and magnetic field, which strongly affects the streamlines. 


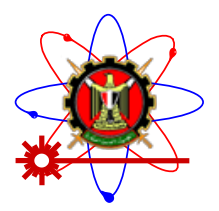

$8^{\text {th }}$ International Conference on Mathematics and Engineering Physics (ICMEP-8)

\section{References}

[1]Latham, T. W.,"Fluid motions in peristaltic pump", MS Thesis, MIT, Cambridge, Massachussetts, 1966.

[2]Fung, Y. C. and Yih, C. S., "Reynolds number and wavelength in peristaltic motion of moderate amplitude ", Journal FluidMechanics, 37, 575-579, 1969.

[3] Shapiro,A.H., Jaffrin, M.Y., and Weinberg, S.L.,"Peristaltic pumping withlong wavelength at low Reynolds number", Journal FluidMechanics, 37, 799-825, 1969.

[4]Agarwall, H. L. and Anwaruddin, B., "Peristaltic flow of blood in a branch", RachiUniversity Mathematics Journal, 15, 108-111, 1984.

[5] Ravikumar, S., Prabhakar, D., Kathyayani, A.,SivaPrasad, R. andPrabhakaraRao, G., $\quad$ "Peristaltic flow of a dusty couple stress fluid in a flexible channel", International Journal OpenProblems ComputerMathematics, 3, 112-116, 2010.

[6] Reddy,G. R. and Venkataramana, S.,"Peristaltic transport of a conducting fluid through a porousmedium in an asymmetric vertical channel",Journal of Advances in Applied Sciences, 2, 240-248, 2011.

[7] Jain,M.,Sharma,G. C. and Singh,A.,"Mathematical analysis of mhd flow of blood in very narrowcapillaries", International Journal of Engineering, 22, 307-315, 2009.

[8]Ravikumar,S., PrabhakaraRao, G. and Siva Prasad, R.,"Peristaltic flow of a couple stress fluidsflows in a flexible channel under an oscillatory flux", International Journal of Applied Mathematics and Mechanics, 6, 58-71, 2010.

[9] RaghunathaRao, T. and Prasad,DRV.,"Peristaltic flow of a couple stress fluid through porous medium in a channel at low Reynolds number", International Journal of Applied Mathematics and Mechanics 8, 97-116, 2012.

[10] Mishra,M.andRamachandraRao, A., "Peristaltic transport of a Newtonian fluid in an asymmetric channel", ZAMP, 53, 530-532, 2003.

[11] Sobh, A. M., Al Azab, S. S. and Mady, H. H.,"Heat Transfer in Peristaltic flow of Viscoelastic fluid in an asymmetric channel", Applied Mathematics Sciences, 4, 1583-1606, 2012.

[12] RaghunathaRao, T. and Prasad, D. V.,"Peristaltic flow of a couple stress fluid through porous medium in a channel at low Reynolds number", 
Military Technical College

Kobry Elkobbah,

Cairo, Egypt

April 19-21,2016

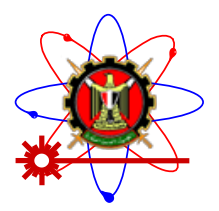

$8^{\text {th }}$ International Conference on Mathematics and Engineering Physics (ICMEP-8)

International Journal of Applied Mathimatics and Mechanics, 8, 97-116, 2010.

[13] Mekhemer, Kh. S., Al-Arabi, T. H., "Non-linear peristaltic transport of MHD flow through porous medium", International Journal Mathematics Sciences, 26 , 1660-1663, 2003.

[14] Abd El Hakeem, Abd El Naby, ElMisery A. M., Abd El Kareem, M. F."Effects of a magnetic field on trapping through peristaltic motion for generalize Newtonian fluid in a channel", PhysicaActa,367, 76-79, 2006.

[15] Eldabe, N. M., El-Sayed, M. F., Ghaly, A. Y. and Sayed, H. M., "Peristaltically induced transport of a MHD biviscosity fluid in a nonuniform tube", PhysicaActa, 383, 253-266, 2007.

[16] RamanaKumari, A. V. and Radhakrishnamacharya, G., "Effect of slip on peristaltic transport in an inclined channel with wall effects", International Journal of Applied Mathematics and Mechanics, 7, 1-14, 2011.

[17] Eldesoky,I. M., "The slip effects on the unsteady MHD pulsatile blood flow through porous medium in an artery under the effectof body acceleration", International Journal of Mathematics and Mathematical Sciences, 2012, 126, 2012.

[18] El-Shehawy, E. F.,El-Dabe, N. T. and El-Desoky, I. M.,"Slip effects on the peristaltic flow of a non-Newtonian Maxwellian fluid",ActaMechanica, 186,141-159, 2006.

[19] Eldesoky,I. M., "Influence of slip condition on peristaltic transport of a compressible Maxwell fluid through porous medium in a tube",International Journal of Applied Mathematics and Mechanics, 8, 99-117, 2012.

[20] Ali,N.,Hussain,Q.,Hayat,T., and Asghar,S., "Slip effects on the peristaltic transport of MHD fluid with variable viscosity",Physics Letters A, 372, 1477-1489, 2008.

[21] Sreenadh, S., Uma Shankar, C. and Raga Pallavi, A., "Effects of wall properties and heat transfer on the peristaltic transport of food bolus through oesophagus-A Mathematical Model",International Journal of Applied Mathematics and Mechanics, 8, 93-108, 2012.

[22] Medhavi, A. and Singh, U. K., "A two-layered suspension flow induced byperistaltic waves", International Journal Fluid Mechanics Research, 35, 258-272, 2008.

[23] Mekheimer, Kh. S., "Peristaltic motion of a particle-fluid suspension in a planarchannel", International Journal Theory Physics, 37, 2895-2920, 1998. 
Military Technical College Kobry Elkobbah,

Cairo, Egypt

April 19-21,2016

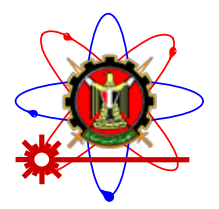

$8^{\text {th }}$ International Conference on Mathematics and Engineering Physics (ICMEP-8)

[24] Srivastava, V.P., "Particle-fluid suspension flow induced by peristaltic waves ina circular cylindrical tube", Bull. Calculation Mathematics Society, 94, 167-184, 2002.

[25] Kamel, M. H., Eldesoky, I. M., Maher, B. M. and Abumandour, R. M., "Slip effects on peristaltic transport of a particle fluid suspension in a planar channel", Applied Bionics and Biomechanics, 2015, 1-14, 2015. 